\title{
Drag and lift forces on clean spherical and ellipsoidal bubbles in a solid body rotating flow.
}

\author{
Marie Rastello, Jean-Louis Marié, Michel Lance \\ Laboratoire de Mécanique des Fluides et d'Acoustique \\ Ecole Centrale de Lyon - CNRS - Université Claude Bernard Lyon 1 - INSA Lyon \\ 36 avenue Guy de Collongue, 69134 Ecully cedex, France
}

(Received )

A single bubble is placed in a solid body rotating flow of silicon oil. From the measurement of its equilibrium position, lift and drag forces are determined. Five different silicon oils have been used, providing five different viscosities and Morton numbers. Experiments have been performed over a wide range of bubble Reynolds numbers $(0.7 \leq R e \leq$ $380)$, Rossby numbers $(0.58 \leq R o \leq 26)$ and bubble aspect ratios $(1 \leq \chi \leq 3)$. For spherical bubbles, the drag coefficient at the first order is the same as that of clean spherical bubbles in a uniform flow. It noticeably increases with the local shear $S=R o^{-1}$, following a $R o^{-5 / 2}$ power law. The lift coefficient tends to 0.5 for large $R e$ numbers and rapidly decreases as $R e$ tends to zero, in agreement with existing simulations (Magnaudet and Legendre 1998). It becomes hardly measurable for $R e$ approaching unity. When bubbles start to shrink with $R e$ numbers decreasing slowly, drag and lift coefficients instantaneously follow their stationary curves versus Re. In the standard Eötvös Reynolds diagram, the transitions from spherical to deformed shapes slightly differ from the uniform flow case, with asymmetric shapes appearing. The aspect ratio $\chi$ for deformed bubbles increases with the Weber number following a law which lies in between the two expressions derived from the potential flow theory by Moore $(1959,1965)$ at low and moderate $W e$, and the bubble orients with an angle between its minor axis and the direction of the flow that increases for low $R o$. The drag coefficient increases with $\chi$, to an extent which is well predicted by the Moore (1965) drag law at high $R e$ and $R o$ numbers. The lift coefficient is a function both of $\chi$ and $R e$. It increases linearly with $(\chi-1)$ at high $R e$ in line with the inviscid theory, while in the intermediate range of Reynolds numbers, a decrease of lift with aspect ratio is observed. But the deformation is not sufficient for a reversal of lift to occur.

\section{Introduction}

Bubbles are present in many industrial and environmental flows (oil industry, chemical engineering, water purification etc...). The importance of understanding their behavior is obvious. A central question for a number of applications is to predict where bubbles are distributed and what the consequences are on the local transfers (momentum, heat, mass). When the flow is non-homogeneous, one physical effect that influences the preferential migration and accumulation of bubbles is the transverse (or lift) force acting on them. The mechanism by which the lift is produced depends on the Reynolds number built on the velocity of bubbles relative to the flow, and the bubble's diameter. This has motivated researchers to study the forces on bubbles by considering different shear flow configurations. The case of spherical bubbles in linear shear flow is now well described. For instance, Legendre and Magnaudet (1998) computed the drag and lift coefficients over a wide range of Reynolds numbers and shear rates and provided information on the physical mechanisms involved in the lift force. The effect of bubble deformation had also been investigated to some extent . Kariyasaki (1987), Ervin and Tryggvason (1997), Tomiyama et al. (2002), Adoua et al. (2009) found that this deformation can generate a reversal of the lift, which would explain the transition from void wall peaking to void coring observed in pipe flows (Serizawa et al. 1975).

In comparison, other interesting situations regarding transverse forces have been less extensively covered. Solid rotation flow is one of them. Its main difference with shear flow is that it does not include any strain effect, hence is more representative of regions with local vorticity (e.g. mixing layers). The works available in the literature can be categorized into solid body rotation around vertical and horizontal axes (see Bluemink et al. (2008), Rastello et al. (2009) for a survey). Experimentally, the main advantage of solid body rotation around an horizontal axis (SBRH) is that bubbles reach an equilibrium point, from which it is possible to measure the drag and lift coefficients. Moreover, since the bubble is kept stationary, interface deformation and interface contamination, both crucial for the forces, can be more easily investigated. On the purity of the bubble surface will depend the boundary conditions to which 
the surrounding liquid is subjected. When the bubble is clean, the liquid is subjected to a shear-free condition and free to slip along the surface. When the interface is contaminated, this boundary condition is no longer satisfied, so that the slip of the liquid at the bubble surface can be reduced or even suppressed. The forces acting on the bubble in both cases will obviously be different. The numerical study that makes reference for this type of flow is that by Magnaudet and Legendre (1998). It concerns a clean spherical bubble kept stationary in a SBRH. The lift coefficient was computed over a wide range of Reynolds numbers $(0.1 \leq R e \leq 1000)$ and the transition between viscous and inviscid behavior analysed. The influence of the shear on the drag coefficient was also quantified. The experimental works were performed with nearly spherical bubbles in rotating tanks filled either with a mixture of water and glycerine (Naciri 1992; van Nierop et al. 2007), or with demineralized water (Rastello et al. 2007, 2009). They showed that the lift coefficients may significantly differ from those expected numerically for clean bubbles, indicating that some degree of contamination may have been present. Naciri (1992) was the first to run this type of experiment. He worked with slightly deformed, probably contaminated bubbles, and measured a lift coefficient of the order of 0.2 to 0.3 , for $R e$ numbers ranging from 10 to 100. van Nierop et al. (2007) revisited Naciri's experiment by extending the range of local shear rate $(0.1<S<2)$ and bubble Reynolds number $(0.01<R e<500)$. The drag curves reported are close to those of a solid sphere, indicating that interfaces were probably not perfectly clean. The lift coefficients found at high $R e$ are typically around 1, rather than the value 0.5 expected by the simulation (Magnaudet and Legendre 1998) or the inviscid theory (Auton 1987), while, quite remarkably they become negative at low Reynolds numbers $(R e<5)$ with increasing viscous effects. Recently, we made measurements with highly contaminated interfaces (Rastello et al. $2007,2009)$ over an intermediate range of Reynolds numbers $(4<R e<280)$. The low- $R e$ case was not investigated and so the negative lift was neither confirmed nor refuted. It was shown that when interfaces are contaminated the flow at the interface spins around the bubble, inducing an additional lift component due to a Magnus-like effect that adds to the shear induced lift. We used our measured spin rates in combination with the Magnus-like force proposed by Bagchi and Balachandar (2002) to predict the increase of lift due to this effect. Similar results have been reported by Bluemink et al. (2010) for spinning solid spheres.

The objective of the present experiment is to complete our previous study by investigating a situation where the interfaces are clean. We thus want to confirm that the higher lift coefficients reported in the intermediate and high Reynolds range come from contamination. Attention will be also focused on low Reynolds numbers where the lift sign can be expected to change. Finally, our intention is to investigate the effect of bubble deformation on the drag and lift coefficients and see whether lift can be reversed,like in linear shears under certain conditions. Since it is extremely difficult to avoid contamination by active surface molecules or impurities, as is the case when using polar fluids such as water (Duineveld 1995; de Vries et al. 2002a), we work with five silicon oils, whose physical properties are perfectly calibrated and stable in time. Experiments performed with silicon oils were recently found to be conclusive to check theoretical predictions regarding several aspects of the dynamics of spherical and spheroidal bubbles, e.g. wall interaction as in Takemura et al. (2002), Takemura et al. (2009), or the onset of path instability as in Zenit and Magnaudet (2008). We thus adopt the same strategy for determining the shear-induced lift force. As a result, interfaces can be kept free of contaminants and the drag and lift forces measured by varying the non-dimensional numbers of the problem $(R e, R o$, and $M o$ ), over a wide range of values.

This paper is set out as follows. First, the force balance and the dimensionless numbers are recalled for spherical and ellipsoidal cases. Then, the experimental set-up, measurement principle and flow characteristics are briefly recalled. The drag and lift coefficient measurements are presented and commented on in the two last parts, starting by spherical bubbles and ending with deformed ones.

\section{Force balance, dimensionless numbers}

\subsection{Spherical bubbles}

For a spherical bubble, moving relatively to the fluid at a moderate to large Reynolds number, the dynamical equation commonly adopted to describe the bubble motion when any possible history effects are neglected is (Magnaudet and Eames 2000):

$$
\begin{aligned}
\rho_{l} V_{b} C_{A} \frac{d \mathbf{v}}{d t} & =-\rho_{l} V_{b} \mathbf{g}+\rho_{l} V_{b}\left(C_{A}+1\right) \frac{D \mathbf{U}}{D t} \\
& +\rho_{l} V_{b} C_{L}(\mathbf{U}-\mathbf{v}) \times(\nabla \times \mathbf{U})+\frac{1}{2} \rho_{l} C_{D} A_{b}|\mathbf{U}-\mathbf{v}|(\mathbf{U}-\mathbf{v})
\end{aligned}
$$




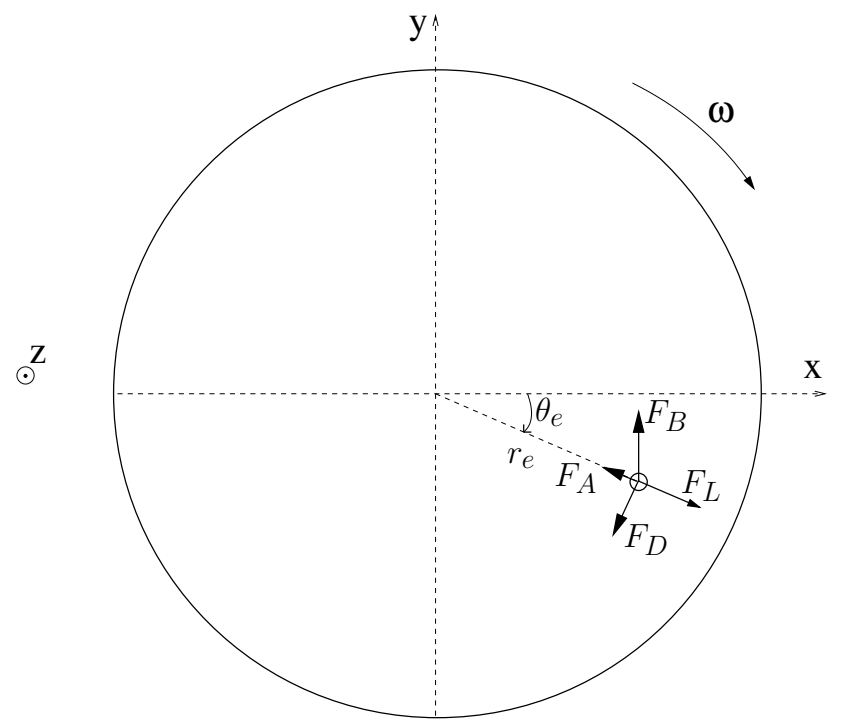

FIGURE 1. The forces applied to a bubble at equilibrium at moderate to high Reynolds numbers (Rastello et al. 2009). $\mathbf{F}_{B}$ is the buoyancy force, $\mathbf{F}_{D}$, the drag force, $\mathbf{F}_{L}$ the lift force. $\mathbf{F}_{A}$ takes into account the added mass force and pressure gradient effects. In this diagram, the angular velocity $\omega$ and equilibrium angle $\theta_{e}$ are negative.

where $\rho_{l}$ is the liquid density, $V_{b}$ the volume of the bubble, $A_{b}$ its projected area, $\mathbf{v}$ the bubble's velocity, $\mathbf{g}$ gravity acceleration and $\mathbf{U}$ the velocity of the undisturbed ambient flow taken at the center of the bubble. The forces considered in this equation are respectively buoyancy $\mathbf{F}_{B}$, added mass and pressure gradient $\mathbf{F}_{A}, \operatorname{lift} \mathbf{F}_{L}$ and $\operatorname{drag} \mathbf{F}_{D} . C_{A}$ is the added mass coefficient. Its value is 0.5 for spherical bubbles (Magnaudet and Eames 2000). $C_{L}$ is the lift coefficient and $C_{D}$ the drag coefficient. In the present experiment the undisturbed flow field $\mathbf{U}$ is a solid body rotating flow at constant angular velocity $\omega$ around a horizontal axis $(S B R H)$. In this case all forces balance when the flow is stationary and the bubble comes to an equilibrium position (see figure 1) where $\mathbf{v}=\mathbf{0}$. With $\mathbf{U}=\omega r \mathbf{e}_{\theta}$, equation (2.1) becomes:

$$
C_{L}=\frac{1}{2}\left(1+C_{A}-\frac{g \sin \theta_{e}}{r_{e} \omega^{2}}\right)
$$

and

$$
C_{D}=\frac{4}{3} \frac{g d \cos \theta_{e}}{r_{e}^{2} \omega^{2}}
$$

where $\left(r_{e}, \theta_{e}\right)$ are the polar coordinates $(r, \theta)$ of the center of the bubble at equilibrium and $d$ the equivalent diameter of the bubble. Thus, if one measures $r_{e}$ and $\theta_{e}, d$ and $\omega$, the values of $C_{L}$ and $C_{D}$ can be calculated. At low Reynolds numbers the force balance cannot be expressed in the form (2.1). The total hydrodynamic force on the bubble at leading-order with respect to Re.S is simply the sum of the Stokes drag and a low-Re lift which accounts for the inertia effects (Saffman 1965; Harper and Chang 1968). This low-Re lift scales as $(R e . S)^{1 / 2}$ and not as Re.S as is the case in (2.1) (Magnaudet and Legendre 1998). Equations (2.2), (2.3) have to be modified accordingly. As the flow is stationary when the bubble is at rest, no contribution of the history force used to describe non-stationary effects in this small Reynolds regime (Candelier et al. 2004; Wakaba and Balachandar 2005) is to be expected in this last stage. The equations for the equilibrium can be used as long as a radial force exists to enable a stationary bubble position, which is no longer the case when Re tends to zero. Indeed, in this limit, the Stokes drag balances the buoyancy and the bubble describes circles at constant angular velocity $\omega$, corresponding to $\mathbf{F}_{B}+\mathbf{F}_{D}=\mathbf{0}$ :

$$
\mathbf{v}=\omega r \mathbf{u}_{\theta}-\frac{d^{2}}{12 \nu} \mathbf{g}
$$

As discussed in Rastello et al. (2009), three non-dimensional numbers are formally needed to describe the flow when working with different fluids. The three numbers chosen here to parametrize the results are: $R e=(v-U) d / \nu$, the Reynolds number based on bubble diameter and on the relative velocity; $R o=(v-U) / \omega d$, the Rossby number that takes into account the non-homogeneity of the rotational flow; $M o=g \nu^{4} \rho^{3} / \sigma^{3}$ the Morton number whose main advantage is to be a function only of the fluid properties. When the bubble is at equilibrium, $R e=\omega r_{e} d / \nu$ and 
$R o=r_{e} / d$. It must be noted that $R o$ in the present arrangement is simply the inverse of the non-dimensional local shear rate $S$, and is used to characterize the effects of the strain and the curvature of the streamlines "seen" by the bubble at rest. Bubbles remain perfectly spherical (aspect ratio $\chi=1$ ) as long as surface tension effects dominate the normal stress. In that case, surface tension can be considered as infinite, which means that the Mo number does not influence this regime. It can therefore be removed from the previous list. This is no longer true as bubbles start to depart from spheres. As will be seen in subsection 5.1, the shape transition takes place more or less early with $R e$, according to the $M o$.

\subsection{Deformed bubbles}

For ellipsoidal bubbles the same force balance can be used (de Vries et al. 2002b). The various coefficients are then a function of the bubble shape. At equilibrium, $C_{D}$ and $C_{L}$ can be determined, as for spherical bubbles, from equations (2.3) and (2.2). We choose to define the drag coefficient in 2.1 by taking the projected area $A_{b}$ of a sphere of identical volume, hence $d$ there denotes the equivalent diameter. The stabilized bubbles being mostly oblate spheroids with their small axis approximately aligned with the flow direction (section 5), $C_{D}, C_{L}, C_{A}$ will depend on the aspect ratio $\chi$. The component of the added mass tensor parallel to the direction of the flow relative to the bubble is equal to $\rho_{l} V_{b} C_{A}$, where $C_{A}$ is given by (Lamb 1934):

$$
C_{A}=\frac{\gamma_{0}}{2-\gamma_{0}} \text { with } \gamma_{0}=\frac{2}{e^{2}}\left(1-\sqrt{1-e^{2}} \frac{\operatorname{asin}(e)}{e}\right) \text { and } e=\frac{\sqrt{\chi^{2}-1}}{\chi}
$$

This expression is valid as far as the bubble can be approximated by an oblate spheroid. As added mass effects result from the equality of the normal liquid and gas velocities at the interface, it is appropriate whatever the flow past the bubble is separating or not. A fourth non-dimensional number is needed to account for the bubble deformation. The aspect ratio $\chi$ characterizes the geometry, more than the physical mechanisms involved in the shape change. Since deformation is linked to the dynamic pressure distribution seen by the bubbles and surface tension effect, the Weber number $W e=\rho(v-U)^{2} d / \sigma=\rho \omega^{2} r_{e}^{2} d / \sigma$ is generally employed, together with the Reynolds number (Clift et al. 1978). However, in experiments on ascending bubbles, this set is often replaced by the Eötvös number Eo $=$ $g \Delta \rho d^{2} / \sigma=g \rho d^{2} / \sigma$ and the Morton number, which are more straightforward to specify. Referring to Loth (2008), buoyancy effects included in Eo are not directly responsible for deformation and, for that reason the two sets are equivalent only in situations of equilibrium, where drag balances buoyancy. This is almost the case here, so that both sets are possible. Because we use different silicon oils, we will keep as for spherical bubbles the Mo and finally retain the set $R e, R o, M o, W e$.

\section{Experimental features}

A complete description of the facility and of the flow field characteristics can be found in Rastello et al. (2007, 2009). It is sufficient to say that the device is a cylindrical tank $(\varnothing=10 \mathrm{~cm}, \mathrm{~L}=10 \mathrm{~cm})$ rotating around a horizontal axis. The tank is made of Plexiglas (see figure 2) and is connected to a motor by a toothed belt. For this set of experiments, the range of rotation rates for the tank is $\left[4 \mathrm{rad} . \mathrm{s}^{-1}, 27 \mathrm{rad} . \mathrm{s}^{-1}\right]$. The fluids used are silicon oils. As specified earlier, the advantage of such fluids is that tensioactive molecules do not systematically collect at the bubble's surface as they would do in water. Hence the bubbles' interface remains clean and bubbles behave like clean bubbles whereas they have a contaminated behavior in water. Five oils with five different viscosities have been used so as to explore a large $R e$ range and different $M o$ numbers. Their main physical properties are listed in table 1 . All the experiments were performed by following the same procedure. The temperature $(T)$ of the fluid contained in the tank is first measured using a thermometer. A bubble is then injected. After that, the tank is set in rotation. Once time necessary for the fluid to reach constant angular velocity and for the bubble to stabilize at its equilibrium has elapsed,images are taken with the Phantom V4.3 high speed camera in front of the disposal. The 2000 images for each run are treated using in-house software under Matlab to give the position of the bubble, its diameter, $R e, C_{D}, C_{L} \ldots$

\section{Spherical bubbles}

\subsection{Working context}

Usually (Clift et al. 1978), bubbles are considered spherical if the minor to major axis ratio lies within $10 \%$ of unity. When stabilized, the bubbles always move slightly around their equilibrium position. The amplitude of these motions 


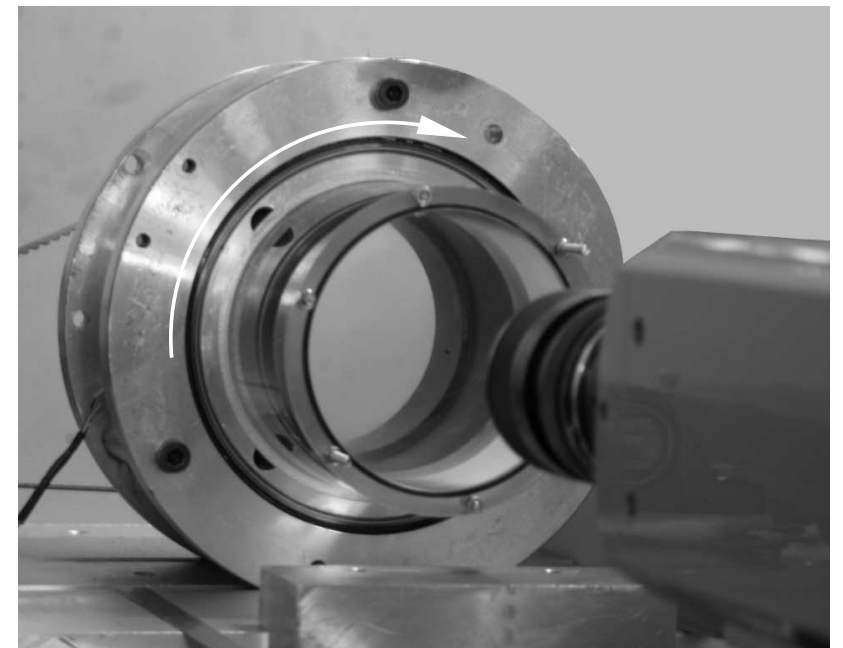

FIGURE 2. Photograph of the experimental device showing the tank (Rastello et al. 2009). In front of it is a high-speed camera. In the back the toothed belt can be seen.

\begin{tabular}{|c|c|c|c|c|c|}
\hline Name & molecular formula & $\nu(\mathrm{cSt})$ & $\alpha$ & $\sigma(\mathrm{mN} / \mathrm{m})$ & $S G$ \\
\hline Rhodorsil@ 47V100 & $\left(\mathrm{CH}_{3}\right)_{3} \mathrm{SiO}\left(\left(\mathrm{CH}_{3}\right)_{2} \mathrm{SiO}\right)_{100} \mathrm{Si}\left(\mathrm{CH}_{3}\right)_{3}$ & 100 & 0.60 & 20.9 & 0.965 \\
\hline Rhodorsil@ 47V20 & $\left(\mathrm{CH}_{3}\right)_{3} \mathrm{SiO}\left(\left(\mathrm{CH}_{3}\right)_{2} \mathrm{SiO}\right)_{20} \mathrm{Si}\left(\mathrm{CH}_{3}\right)_{3}$ & 20 & $|0.59|$ & 20.6 & 0.950 \\
\hline Rhodorsil@ 47V5 & $\left(\mathrm{CH}_{3}\right)_{3} \mathrm{SiO}\left(\left(\mathrm{CH}_{3}\right)_{2} \mathrm{SiO}\right)_{5} \mathrm{Si}\left(\mathrm{CH}_{3}\right)_{3}$ & 5 & 0.55 & 19.7 & $0.910 \mid$ \\
\hline Dow Corning 200ß $1 \mathrm{cst}$ & $\left(\mathrm{CH}_{3}\right)_{3} \mathrm{SiO}\left(\left(\mathrm{CH}_{3}\right)_{2} \mathrm{SiO}\right) \mathrm{Si}\left(\mathrm{CH}_{3}\right)_{3}$ & 1 & $|0.41|$ & 17.4 & 0.818 \\
\hline Dow Corning $200 \AA 0.65 \mathrm{cst}$ & $\left(\mathrm{CH}_{3}\right)_{3} \mathrm{SiOSi}\left(\mathrm{CH}_{3}\right)_{3}$ & 0.65 & 0.31 & 15.9 & 0.761 \\
\hline
\end{tabular}

TABLE 1. Physical properties of the five silicon oils. Kinematic viscosity $\nu$, surface tension $\sigma$ and specific gravity $S G$ are given for

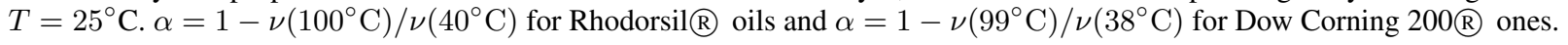

are typically of the order of a tenth of a millimeter, like in water (Rastello et al. 2009). However, the power spectrum of the equilibrium positions in silicon oils is much less noisy and shows that these motions are associated with a single peak at the rotation frequency $\omega$ of the tank. The mean equilibrium values of the parameters $\left(C_{L}, C_{D}, R e \ldots\right)$ are obtained by averaging the instantaneous values over the 2000 images. Although the motions around equilibrium remain small, in certain cases they produce significant oscillations of the instantaneous values (see section 4.4). We have chosen to reject the experiments for which the standard deviation for $C_{D}$ is larger than $30 \%$ of the mean value. The other point that we have checked to be sure of the accuracy of the foregoing measurements is the possible perturbation of the $S B R H$ flow that might result from the bubble's presence. Referring to our previous study in water, no perturbation occurs when the equilibrium position is far from the center $(R o \geq 6)$. In that case, whatever the $R e$, the wake vanishes before a full tank rotation and thus neither impinges on the bubble, nor disturbs the incoming $S B R H$. Conversely, when $R o \leq 6$, a large bubble whose $R e$ is typically $\geq 80$ might become instable with a coriolis-type precession motion at a frequency of $2 \omega$. Strong coupling then develops between the bubble, its wake and the rotational flow, so that the flow seen by the bubble is strongly perturbed. Such experiments must not be retained. Here, no peak at frequency $2 \omega$ were detected on the power spectrum of equilibrium positions. We conclude that this situation is never encountered. 


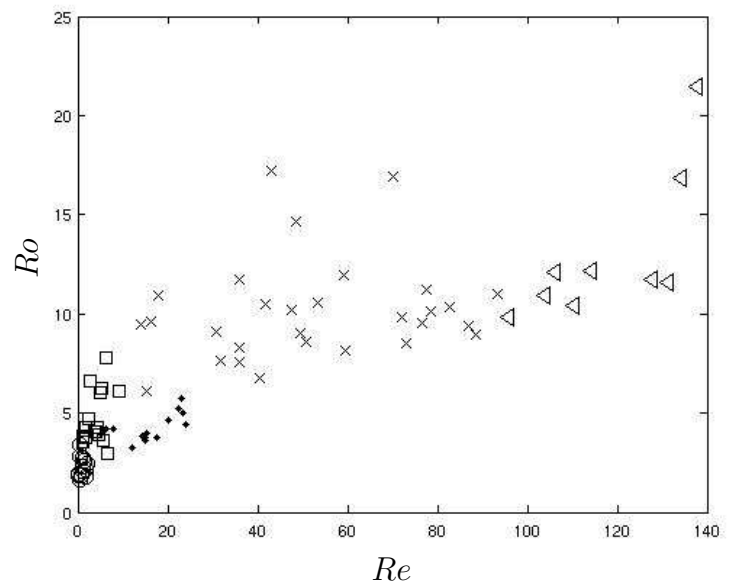

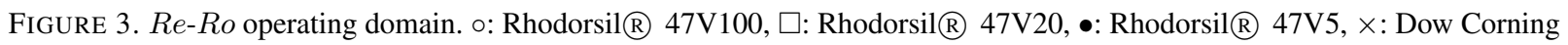
$200 \AA 1$ cst, $\triangleleft$ : Dow Corning 200® 0.65cst.

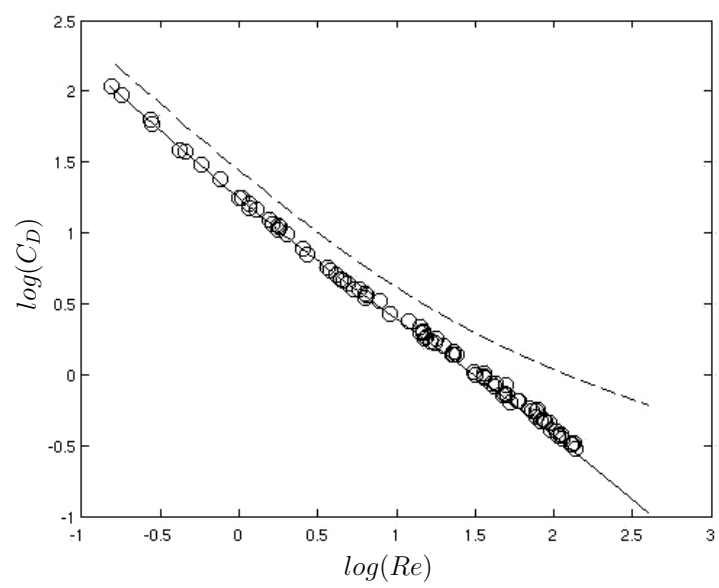

FIGURE 4. $\log \left(C_{D}\right)$ as a function of $\log (R e)$. ॰: present experiments for all the silicon oils; solid line: empirical drag curve for clean bubbles in a uniform flow proposed by Mei et al. (1994) dashed line: drag curve for solid spheres in a uniform flow (Schiller and Nauman 1933 see Clift et al. (1978)). Erratum: these two references (clean bubbles and solid spheres) were reversed by mistake in (Rastello et al. 2009).

Moreover, low Ro numbers ( $R o \leq 6)$ correspond to low $R e$ numbers (see figure 3 ). The bubbles therefore have very thin wakes that dissipate rapidly and do not influence the bubbles. The same conclusions hold for deformed bubbles (section 5).

\subsection{Drag coefficient}

The drag coefficient is first plotted as a function of the Re number in figure 4. The present experimental points fall quite well on the empirical drag law proposed by Mei et al. (1994) for clean bubbles in a uniform flow, which is known to provide an accurate description of the drag law for all $R_{e}$ (Magnaudet and Eames 2000). This contrasts with the previous study performed with similar spherical bubbles, but in water (Rastello et al. 2009). In that case, the experimental points fell on the curve that describes solid spheres in uniform flow, indicating that the bubbles were contaminated. Here, the bubbles appear to have a clean interface. This confirms that, even with small bubbles, the use of silicon oil ensures that a sliding condition can be retained at the bubbles' interfaces. As the flow is not uniform, it is of interest to look at the influence of the Ro number on the drag. For a linear shear flow, the simulations of Legendre and Magnaudet (1998) performed at high $R e$ numbers (300 and 500) show that the drag significantly increases as the non-dimensional local shear rate $S=R o^{-1}$ increases. The increase in that case is mainly due to the modification of the pressure distribution produced by the shear in the front part of the bubble. The value in uniform flow $C_{D(\text { uniform })}$ 


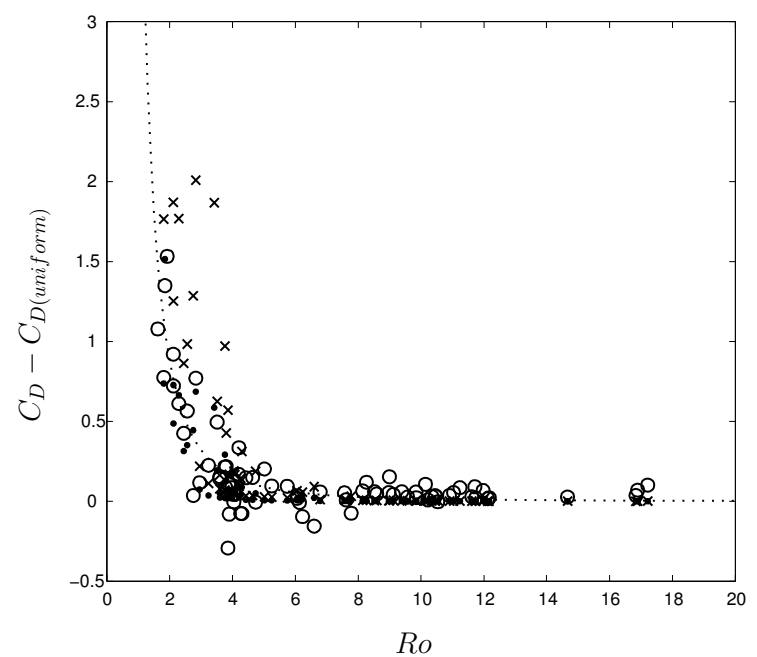

FIgURE 5. $C_{D}-C_{D \text { (uniform) }}$ as a function of $R o . \circ$ : present experiments for all the silicon oils; dotted line:5/Ro $o^{\frac{5}{2}} ; \times$ : equation 4.1 ; equation.: 4.2 .

must be then corrected to take into account this increase. The simple correlation proposed by these authors to fit their results is written as

$$
C_{D}=C_{D(\text { uniform })}\left(1+\frac{0.55}{R o^{2}}\right)
$$

Although the flow is different, our experimental data are compared to this drag law in figure 5. As the data are scattered when divided by $C_{D \text { (uniform) }}$, the comparison was made by plotting the difference $C_{D}-C_{D(\text { uniform) }}$ as a function of $R o$, where $C_{D \text { (uniform) }}$ is the drag coefficient of Mei et al. (1994). In that form, it can be seen that the drag is almost unaffected by the shear rate characterizing the flow rotation for $R o>5$, while it significantly increases for $R o \leq 5$, in agreement with Legendre and Magnaudet (1998). However, the above Ro dependence law tends to slightly underestimate the data in the range which corresponds to the small local shear rates $(\operatorname{Re}>30, R o \in[5 ; 20])$ and significantly overestimate them at high shear rates $(R e<30, R o \in[1 ; 5])$. This is not unexpected in the sense that equation 4.1 accounts for the shear dependence at high Reynolds numbers in a linear shear flow. In our case, besides the fact that the flow is a solid body rotation, the low Rossby numbers $(R o<5)$ or high shear rates correspond to low or moderate Reynolds numbers $(R e<30)$ in the operating plane $(R e, R o)$ investigated, while low shear rates $S<0.15$ are associated with moderate and high Reynolds numbers $R e>30$. This difficulty to vary $R e$ and $R o$ independently, like in the simulations, arises because it is impossible to control the equilibrium position of the bubbles, which is imposed by the bubble's diameter and the rotation rate. For that reason, the comparison with Legendre and Magnaudet (1998) remains limited as regards its physical interpretation. Over the range explored, the data generally follow a $5 / R o^{\frac{5}{2}}$ law well. The deviations from this average law are linked to the $R e$ variations. The dependence on Reynolds and Rossby numbers has been sought in the form $k C_{D \text { (uniform })} / R^{e x p}$. Optimal proportionality factor and exponent have been determined by a least-square fitting method for all pairs $(R e, R o)$ corresponding to the range $R o \in[1 ; 22]$. This yields the following dependence relationship

$$
C_{D}=C_{D(\text { uniform })}\left(1+\frac{0.3}{R o^{\frac{5}{2}}}\right)
$$

Clearly, this drag law reproduces rather satisfactorily the significant increases of drag which are observed for the various $R e$ at low Ro. Unlike equation 4.1, it is established for low, moderate and high Reynolds numbers.

\subsection{Lift coefficient}

The lift coefficient is plotted versus $R e$ number in figure 6. Our data are compared with the results from the numerical simulations of Magnaudet and Legendre (1998). Both fall onto the same curve, unlike in Rastello et al. (2009), where the measured lift coefficients were systematically higher than those calculated. The good agreement between the present data and the simulations is consistent with the previous results on drag. It confirms that the interfaces are 

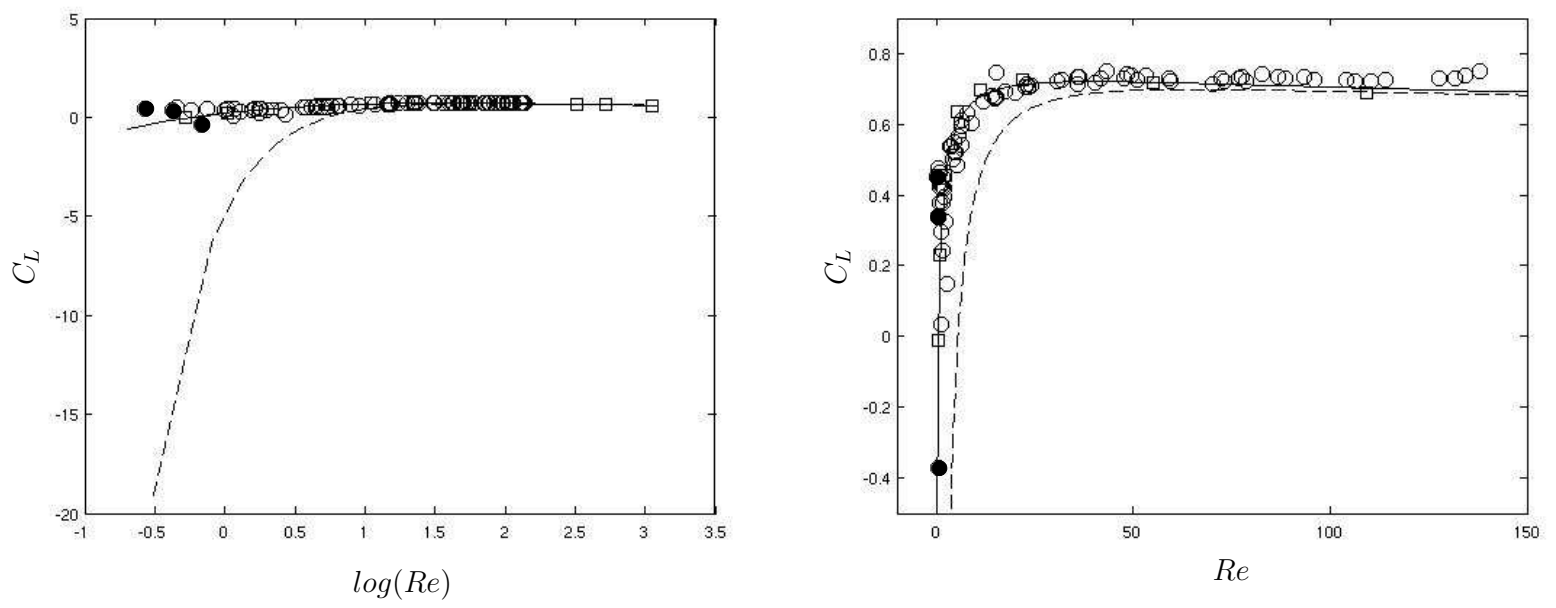

FIGURE 6. Left: $C_{L}$ as a function of $\log (R e)$. Right: $C_{L}$ as a function of $R e$. o: present experiments for all the silicon oils; •: present experiments with circular trajectories; $\square$ : Magnaudet and Legendre (1998) numerical points; solid line: $0.5+4\left(1-6 /\left(5 R e^{\frac{1}{6}}\right)\right) \exp \left(-R e^{\frac{1}{6}}\right) ;$ dashed line: $0.5+1.2 R e^{-\frac{1}{3}}-6.5 R e^{-1}$

clean, so that the boundary condition is of the "shear-free" type as in the calculation. It brings the expected confirmation that the higher values obtained in water by Rastello et al. (2009) with the same experimental procedure are neither imputable to some errors in the measurements nor to the additional micronic fluorescent particles used for the visualizations, but result from the initial contamination of the interface which modifies the boundary conditions. As recalled in the introduction, the quasi non-slip condition existing in this case makes a thin layer of fluid spin around the contaminated bubble interface, increasing lift. Both experimental and numerical points in the figure appear to be well fitted by Magnaudet and Legendre (1998)'s correlation for $R e>50$ :

$$
C_{L}=0.5+1.2 R e^{-\frac{1}{3}}-6.5 R e^{-1}
$$

However this correlation does not fit experimental and numerical data below 50, so we propose another expression that works well for both the high and low Re numbers covered in this study:

$$
C_{L}=0.5+4\left(1-\frac{6}{5 R e^{\frac{1}{6}}}\right) \exp \left(-R e^{\frac{1}{6}}\right)
$$

It is noted that equation 4.4 asymptotically behaves like equation 4.3 for $R e>150$. Magnaudet and Legendre (1998) observed no Ro dependence for the lift coefficient. Here also we are not able to exhibit any Ro dependence for the lift coefficient. If there is one, it should be so small that it will fall within the dispersion of our points. Moreover, if it exists, $R o$ dependence would be much smaller than that observed for contaminated bubbles, where $C_{L}$ was found to increase by a factor of about 1.2-1.4 with $R o$, when $R e$ is greater than 70 .

\subsection{Considerations on the low-Reynolds-number regime}

Because $R e=\omega r_{e} d / \nu$, different conditions are required to achieve an experiment with a low Reynolds number. A fluid with high viscosity is evidently better suited, together with a small diameter for the bubble. Also, the fluid velocity at the stabilization point $\omega r_{e}$ must be small. Unfortunately this velocity is controlled by the two previous parameters $(\nu, d)$, hence, $\omega$ and $r_{e}$ cannot be varied independently. This is shown by eliminating angle $\theta$ between equations 2.2 and 2.3 (Bluemink et al. 2010). If the drag coefficient is estimated by $C_{D}=\frac{16}{R_{e}}$, the equilibrium position $r_{e}$ for very high viscosity and very low viscosity fluids can respectively be approximated by

$$
\begin{gathered}
\frac{r_{e}}{d}=\frac{g d}{3 \nu \omega} \\
\frac{r_{e}}{d}=\frac{g}{d\left(C_{L}-1-C_{A}\right) \omega^{2}}
\end{gathered}
$$

Therefore, increasing (resp. decreasing) $\omega$ will decrease (resp. increase) $r_{e}$. For rotation to be stable, the tank needs 


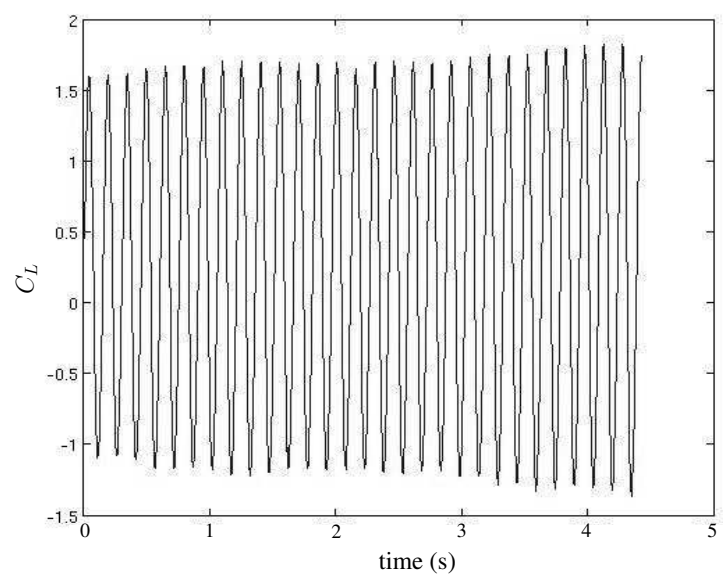

FIGURE 7. Variations of $C_{L}$ with time during a run. $R e=1.9$

to turn at least faster than $4 \mathrm{rad} . \mathrm{s}^{-1}$. Owing to this lower limit, the only possibility to obtain data at low Reynolds numbers, even for the highest fluid viscosity, is to decrease $d$. Hence, as we decrease $R e, r_{e}$ decreases. Such a decrease is problematic for the measurements. As mentioned earlier, bubbles always move slightly around their equilibrium position. The motions are typically of the order of a tenth of a millimeter and generally have a negligible influence on the calculation of the mean quantities. This is no longer true at very low Reynolds numbers where $C_{L}$ tends steeply to zero (figure 6). In that case, the relative importance of these slight motions rises, due to the concomitant decrease of $y_{e}$ and of $r_{e}$ that results from the particular position of the equilibrium point (near the center and the horizontal x-axis). The instantaneous values of $R e, C_{D}$ and $C_{L}$ then begin to exhibit significant oscillations. Their amplitudes remain reasonable (less than $30 \%$ of the mean) for $R e$ and $C_{D}$, but become higher and higher for $C_{L}$ as $R e$ decreases (see for instance figure 7). For $R e \lesssim 3-5$, the amplitude of the oscillations of $C_{L}$ is such that we can wonder whether the mean coefficient calculated from the equilibrium position is still meaningful. This more especially concerns the negative values of the lift coefficient. Just as van Nierop et al. (2007) observed a change of lift sign for Re around 5, we here measure negative values of $C_{L}$ (on average) when $R e$ is $\leq 1$. If we refer to the low-Re theory established by Gotoh (1990), the lift force on a solid sphere kept fixed in a pure solid-body rotation can be expected to change sign at low Reynolds numbers. According to van Nierop et al. (2007), p.444-445, this reversal of lift can also be expected for clean spherical bubbles, where it can be explained in terms of two competing mechanisms. One, centrifugal, is the counterpart of the Saffman shear-induced force for solid spheres. The other, centripetal, is a force generated by the curvature of the wake induced by the rotational flow. This second mechanism, that does not exist in a linear-shear flow (Legendre and Magnaudet 1998), becomes dominant below a certain Re, yielding the change of lift sign. This theory is confirmed for a clean bubble by the few DNS results obtained by Magnaudet and Legendre (1998) over that low- $R e$ range (see figure 6). In a sense, the negative lift coefficients measured here below $R e \simeq 1$ are consistent with all these predictions and the trend provided by fit 4.4. However for the reason mentioned earlier, we can doubt their relevancy. This is all the more true as the use of equation 2.2 and equation 2.3 to determine the force coefficients becomes questionable over this range of $R e$. This idea is reinforced by the nature of the trajectories of very small bubbles. As an example, the trajectory of a bubble whose lift coefficient is negative $\left(R e=0.7, C_{L}=-0.38\right)$ is shown in figure 8. For these small Re numbers, the bubble describes circles with a small amplitude, that compares rather well with the circle (in black) simulated when only the drag force balances the buoyancy force (equation 2.4). Most of these bubbles have been ignored when plotting the results. Nevertheless, three of them are still present in the figures. They are represented by filled circles in figure 6 . Of course, as the low-Re are associated to bubble positions close to the axis, one can also suspect that bubbles are disturbed by their own wake and so are not really embedded in a pure $S B R H$ flow. But at these $R e \leq 1$ (bubble sizes typically $\leq 1 \mathrm{~mm}$ ), the wake is too thin and vanishes too rapidly to significantly influence the flow (see PIV measurements in Rastello et al. (2009)). Therefore, the measurement of the lift becomes very controversial within this limit and it is difficult to conclude if lift reversal does actually take place in our experiment or whether the lift monotonously decreases to zero with the decreasing Reynolds number. If a reversal of lift takes place, the results show that it must occur at very low Reynolds numbers, lower than those reported in the experiment of van Nierop et al. (2007), where some contamination may have been present. Clearly, 


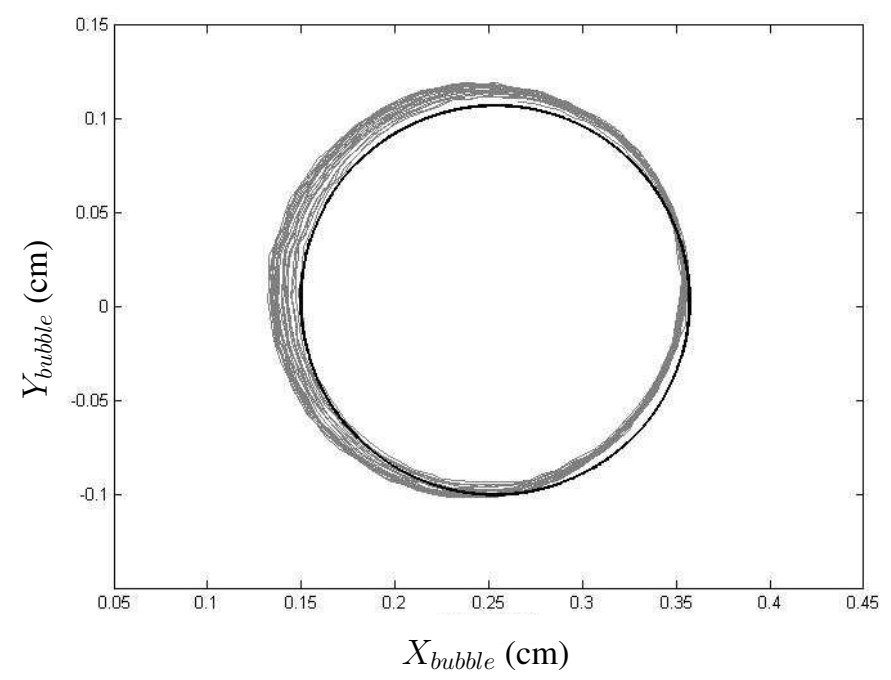

FIGURE 8. In gray: trajectory of a bubble with $R e=0.7, C_{L}=-0.38$. In black: simulated trajectory for the same bubble with only buoyancy and drag forces $\left(C_{A}=C_{L}=0\right)$.

an experiment where the $R e$ number can be varied independently of the bubble position would probably be a more appropriate situation to validate the available low- $R e$ theories and simulations.

\subsection{Drag and lift for shrinking bubbles}

Bubbles in certain silicon oils shrink relatively rapidly compared to bubbles in demineralized water. So, by increasing the duration of the recorded sequences, it is possible to track a bubble which is contracting and to study the time evolution of the forces acting on it. The oil used for this part of our work is Rhodorsil $® 47 \mathrm{~V} 5$, with bubble diameters in the range of $0.2 \mathrm{~mm}-1.5 \mathrm{~mm}$. The diameter of these bubbles decreases linearly with time. The average shrinking speed is typically of the order of $10^{-3} \mathrm{~cm} . \mathrm{s}^{-1}$. Once the bubble introduced into the tank has reached an equilibrium position, it is filmed at a low framing rate for about $75 \mathrm{secs}$. As the bubble starts to contract, the initial equilibrium position drifts slowly toward the axis of the $S B R H$, as expected. A typical example of the instantaneous drag and lift coefficients calculated from the successive positions are plotted versus the instantaneous Reynolds number $R e(t)$ in figures 9 (a) and (b). As can be seen, both coefficients vary in time by following respectively the drag curve of Mei et al. (1994) and the extended lift correlation 4.4 (dotted lines). This means that the contracting bubbles drift in a quasi-static manner, by experiencing at each instant the same force balance and by adopting the same equilibrium position as a constant diameter bubble having the same $\operatorname{Re}(\mathrm{t})$ would do. This result can be explained by the fact that the characteristic time scale of the shrinking process is here much greater than the dynamical relaxation time of the bubble. Interestingly, the lift coefficient measured in figure 9 (b) for the lower Reynolds number $\operatorname{Re}(t)=1.8$ is of the order of that found in figure 6 and is positive around 0.2. This confirms that the reversal of lift, if it takes place, should occur lower.

\section{Deformed bubbles}

\subsection{Shape transitions}

The $R e-R o$ operating domain for deformed bubbles is displayed in figure (10). For bubbles ascending in a uniform medium, the transition between the different shape regimes (spherical, ellipsoidal, wobbling...) can be correlated in terms of Eo-Re-Mo, by the well-known diagram proposed by Clift et al. (1978). The point here is to see whether this diagram is modified in the context of the SBRH. This series of experiments was performed with the silicon oils previously used, but with slightly larger bubbles. Increasing the size yields different shapes depending on silicon viscosity. In low-viscosity oils (Dow Corning 200® 1 cst and $0.65 \mathrm{cst}$ ), bubbles become ellipsoidal before taking on an asymmetric shape at high deformation rates. In the intermediate viscosity range (Rhodorsil@ $47 \mathrm{~V} 5$ and $47 \mathrm{~V} 20$ oils), the same type of transition is observed, the difference being that the bubbles are not perfectly ellipsoidal at moderate deformation rates. However, the deviations are very small and bubbles can reasonably be assimilated to 


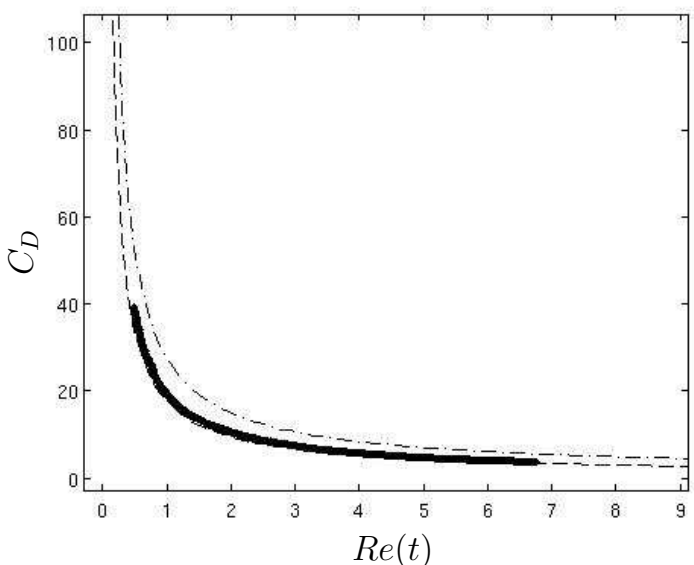

(a)

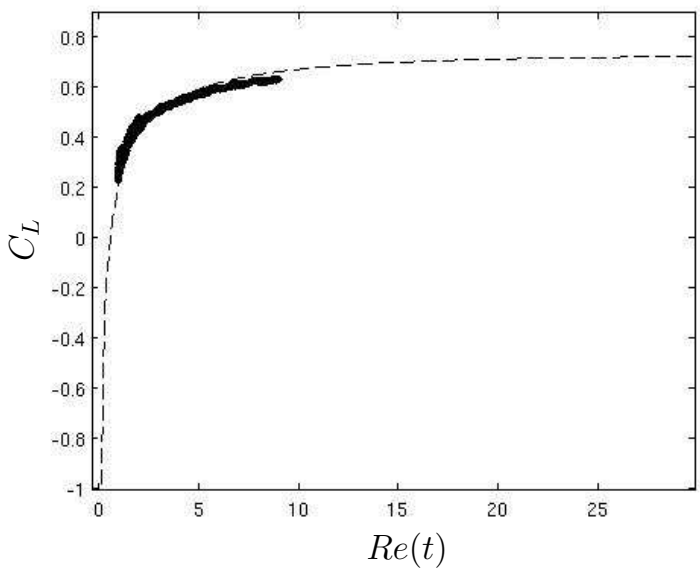

(b)

FIGURE 9. (a): Drag coefficient as a function of the instantaneous $\operatorname{Re}(t)$ number. - - drag curve for clean bubbles in a uniform flow (Mei et al. 1994); - . : drag curve for solid spheres in a uniform flow (Schiller and Nauman 1933 see Clift et al. (1978)); Thick line: evolution of $C_{D}$ with $R e$ for a shrinking bubble. (b): Lift coefficient as a function of the Re number. - - : $0.5+4\left(1-6 /\left(5 R e^{\frac{1}{6}}\right)\right) \exp \left(-R e^{\frac{1}{6}}\right)$; Thick line: evolution of $C_{L}$ with $R e$ for a shrinking bubble.

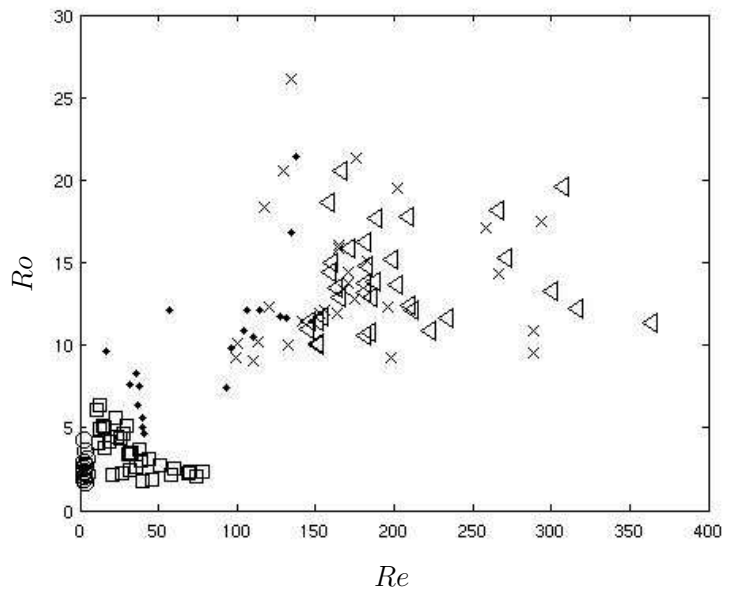

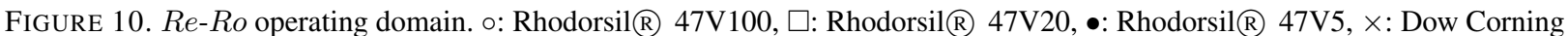

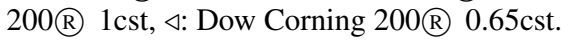

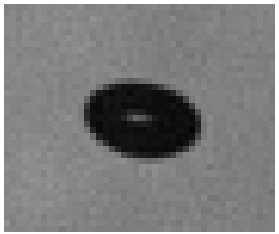

(a)

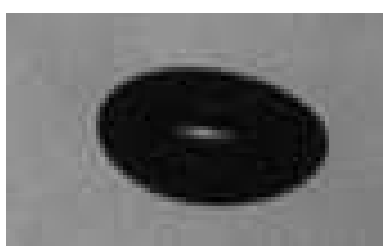

(b)

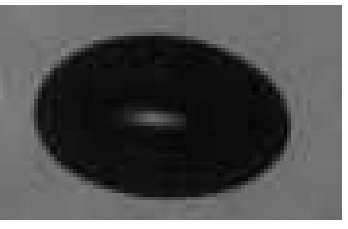

(c)

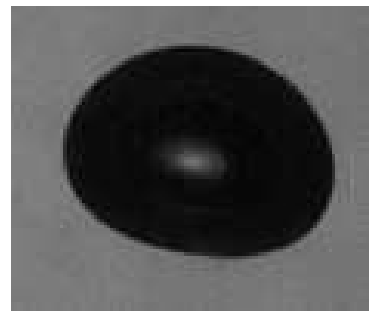

(d)

FIGURE 11. Examples of typical deformed bubbles. Bubbles in the following fluids: (a): Dow Corning 200® 1 cst and 0.65 cst $(R e=331$, major axis length: $1.1 \mathrm{~mm}$, minor axis length: $0.7 \mathrm{~mm}$, equivalent diameter: $0.9 \mathrm{~mm})$, (b): Rhodorsil $\mathbb{R}$ 47V5 $(R e=84$, major axis length: $3.0 \mathrm{~mm}$, minor axis length: $1.8 \mathrm{~mm}$, equivalent diameter: $2.3 \mathrm{~mm}$ ), (c): Rhodorsil@ $47 \mathrm{~V} 20$ $(R e=25$, major axis length: $3.9 \mathrm{~mm}$, minor axis length: $2.5 \mathrm{~mm}$, equivalent diameter: $3.1 \mathrm{~mm})$, (d): Rhodorsil $\AA 47 \mathrm{~V} 100$ ( $R e=4$, major axis length: $5.1 \mathrm{~mm}$, minor axis length: $3.9 \mathrm{~mm}$, equivalent diameter: $4.5 \mathrm{~mm}$ ) 

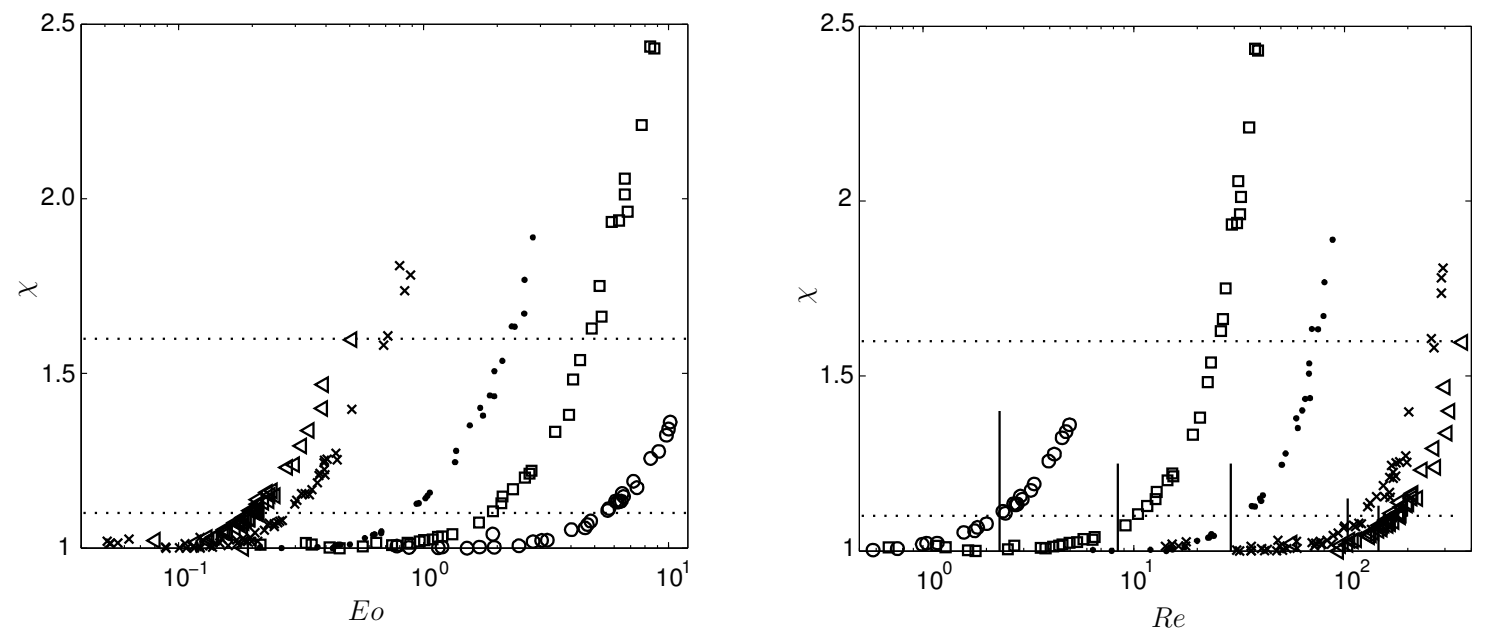

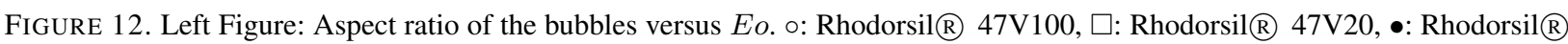
47V5, $\times$ : Dow Corning 200® 1cst, $\triangleleft$ : Dow Corning 200® 0.65cst. Dotted lines: values of $\chi$ corresponding to the shape transitions. Right Figure: Aspect ratio of the bubbles versus Re, same symbols. Straight lines: equation 5.1 and equation 5.2 for the most viscous oil

oblate spheroids. In Rhodorsil $($ 47V100, the first deformed stage is an asymmetric one, more curved at the top and flatter at the rear (see figure 11 for all). For asymmetric shapes an equivalent ellipsoid is computed to determine the equivalent aspect ratio $\chi$. The bubble is fitted using a bivariate normal distribution that has the same normalized second central moment (covariance matrix) as the bubble in the picture (Teague 1980). The directions and lengths of the ellipsoid's axes are given by the eigenvectors and eigenvalues of the covariance matrix of this distribution.

The aspect ratios $\chi$ of the bubbles increase with Eo all the more rapidly as viscosity is low, or Mo number is high (see left figure 12). The Re number for which the deformation becomes significant $(\chi \geq 1.1)$ increases with decreasing viscosity (increasing $M o$ number), following the trends reported in the literature on uniform flow (Clift et al. (1978); Loth (2008)). Moore (1965) calculated the theoretical deformation of clean bubbles in a uniform flow at high Reynolds numbers, by considering the potential flow around an oblate ellipsoid and assuming that the Bernoulli condition, including surface tension effects, is satisfied at both stagnation points and on the equatorial plane. The expression obtained is valid for bubbles whose $W e$ are of the order of unity, which is the condition for their shape could be approximated by an oblate ellipsoid. Moore (1965) used the expansion of this expression in the limit of small deformations $(\chi \rightarrow 1$, Moore (1959)) to estimate the departure from the spherical shape as a function of the $M o$ number. This departure was arbitrarily taken to be $\chi \geq 1.05$ and the terminal velocity of the bubble estimated on the base of a Levich (1962) drag law, yielding for the transition $R e=1.1 \mathrm{Mo}^{-\frac{1}{5}}$. The same calculation with a shape distortion $\chi=1.1$, as adopted in Clift et al. (1978), yields

$$
R e=1.65 \mathrm{Mo}^{-\frac{1}{5}}
$$

and,

$$
R e=1.45 \mathrm{Mo}^{-\frac{1}{5}}
$$

when the bubble terminal velocity is estimated on the basis of a Stokes drag. As can be seen in right figure 12, the departures from the spherical shape which are experimentally observed are rather well predicted by equation 5.1 , and equation 5.2 for the most viscous oil. This shows that the rotation does not really influence the Re at which deformation starts over the range of parameters investigated. However, the shape regimes are somewhat different from those in uniform flows. This is illustrated in figure 13, where the data are plotted on the Clift et al. (1978) diagram for bubbles in uniform flows.

The values of $\log (M o)$ corresponding to the different silicon oils are, by increasing viscosity: $-9.7,-8.9,-6.1,-3.8$, -0.9. Because bubbles are at equilibrium in a $S B R H$, this diagram should include a third dimension depending for example on $R o$. This has been done using different symbols for different $R o$ ranges in figure 13 . When $M o$ is constant the $R e-E o$ curves are nearly the same for uniform and $S B R H$ flows, indicating that the dynamical equilibrium is barely modified by rotation effects. In particular the values of $(R e, E o)$ for which deformation becomes significant for 


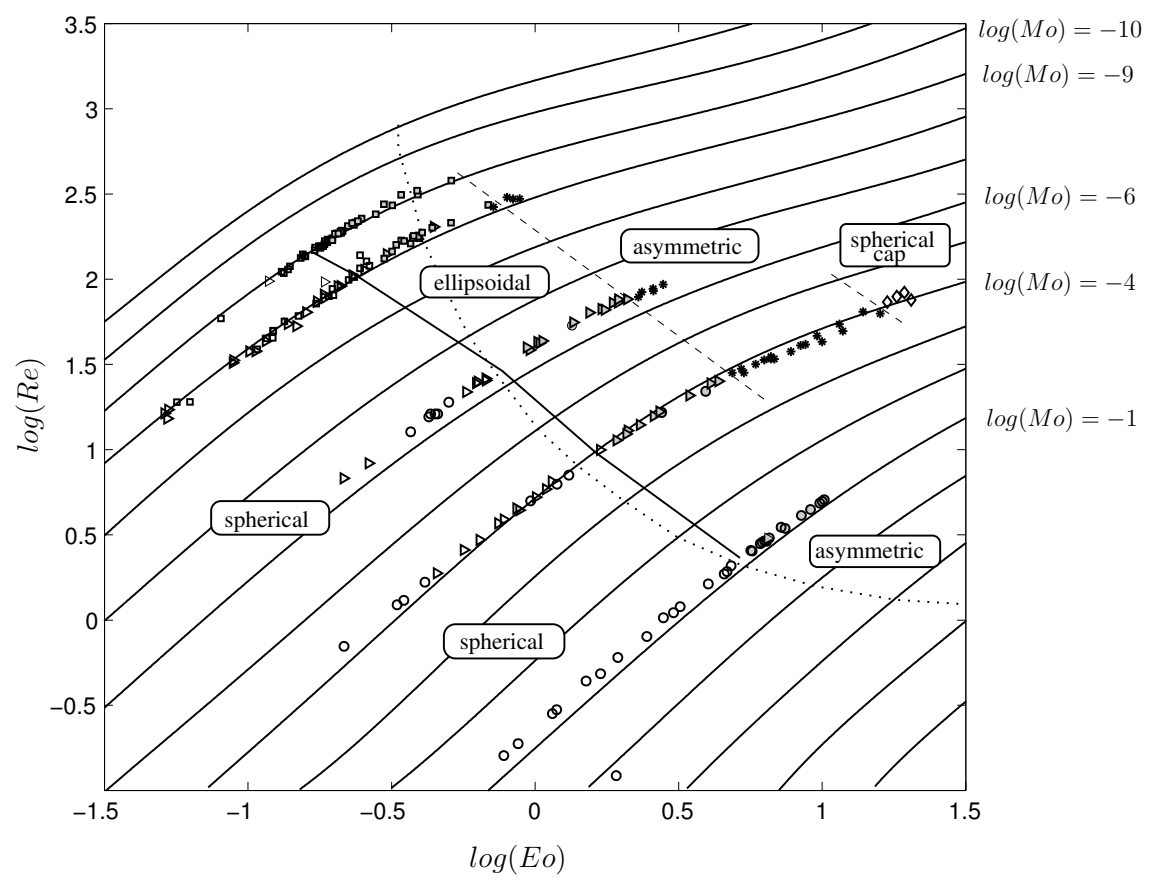

FigURE 13. $\log (R e)$ versus $\log (E o)$. Symbols: $\circ: R o<4, \triangleright: 4 \leq R o<10, \square: 10 \leq R o$. Open symbols: $\chi<1.1$, gray symbols: $1.1 \leq \chi<1.6, *: 1.6 \leq \chi, \diamond$ : spherical cap bubbles. Dotted lines: transition between spherical and ellipsoidal shape according to Grace et al. (1976). Solid line: line joining the transition points between spherical and ellipsoidal shape, as determined by equation 5.1, and equation 5.2 for the most viscous oil. Dashed lines: transition to asymmetric and spherical cap shapes.

the various $M o$ numbers, are very close to the bounds given by equation 5.1, and equation 5.2 in uniform flow, within the precision of our study. Concerning the shape regimes, comparison with the uniform flow situation shows that:

- At small deformation rates $(1.1 \leq \chi<1.6)$, the major change concerns the highest Mo number (the most viscous oil) for which the transition between spherical and ellipsoidal shapes in the uniform flow case is now between spherical and asymmetric shapes. For the two low $M o$ values (least viscous oils), the transition remains unchanged: between spherical and ellipsoidal shapes. For the two intermediate Mo numbers (intermediate viscosities), the deformed shapes are not strictly ellipsoids but slightly asymmetric. These trends can be related to the operating plane (Re, Ro) in figure (10). It is likely that bubbles with low or moderate Reynolds numbers $(R e<30)$ deform asymmetrically because they are associated with low Rossby numbers: $R o \in[1 ; 5]$ (high shear rates), while bubbles with a high Reynolds number $(R e>80)$ remain symmetric because they are subjected to low shear rates. Would low $R e$ have been obtained over that range, it is not certain that this would be the case.

- At high deformation rates $(\chi \geq 1.6)$, a second transition from ellipsoidal or quasi-ellipsoidal to asymmetric bubbles takes place in the domain where, in uniform flow, bubbles with a symmetrical shape are still expected (Grace et al. 1976). The corresponding Rossby numbers are rather high ( $R o \in[4 ; 22])$, but since the $W e$ number is high too, the moderate shear rates which are associated are sufficient to explain these asymmetrical shapes. Finally for the more viscous oil such that with $\log (M o)=-3.8$, the transition between asymmetric bubbles and spherical cap ones is observed.

\subsection{Bubble features}

As specified in section 2.2, we chose to work with $R e, R o, W e$ and $M o$ non-dimensional numbers. The fact that the $W e$ is well suited to describe the bubble deformation is illustrated in figure 14, where the bubble's Reynolds numbers for the different oils collapse when plotted versus $W e^{\frac{2}{3}} M o^{-\frac{1}{5}}$. The corresponding correlation curve is written as:

$$
R e=2.05 W e^{\frac{2}{3}} M o^{-\frac{1}{5}}
$$

which is very similar to the equation derived by Moore (1965) for high $R e$ on the basis of a Levich (1962) drag law

$$
R e=2.04 W e^{\frac{3}{5}} M o^{-\frac{1}{5}}
$$

The aspect ratio $\chi$ is plotted as a function of the $W e$ number in figure 15 , together with the complete expression 


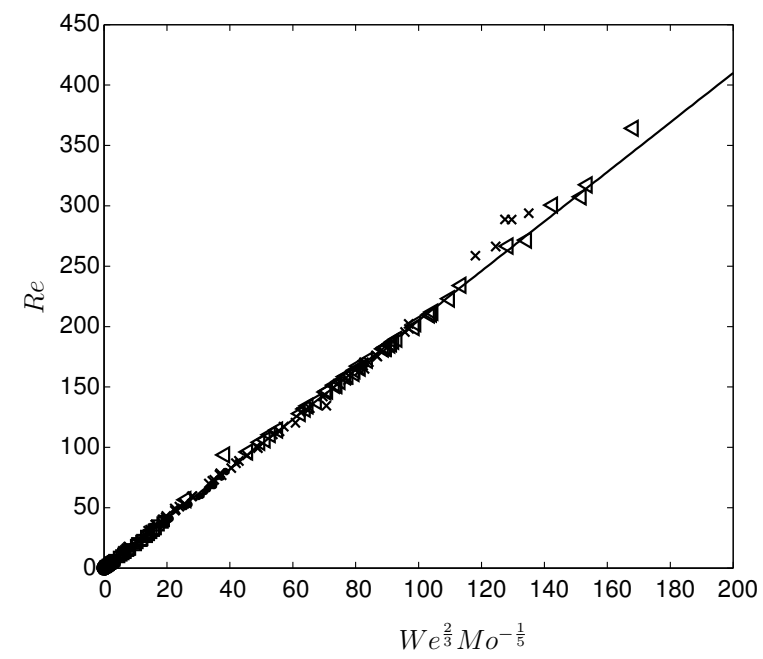

FIgURE 14. Re versus $W e^{\frac{2}{3}} M o^{-\frac{1}{5}}$. ॰: Rhodorsil@ 47V100, $\square$ : Rhodorsil@ 47V20, •: Rhodorsil@ 47V5, $\times$ : Dow Corning $200 \Re 1$ cst, $\triangleleft$ : Dow Corning $200 ® 0.65$ cst. Correlation curve: $2.05 W e^{\frac{2}{3}} \mathrm{Mo}^{-\frac{1}{5}}$.

derived from the potential flow theory by Moore (1965) for We numbers of the order of unity and its expansion at small $W e$, respectively:

$$
\begin{gathered}
W e(\chi)=4 \chi^{-\frac{4}{3}}\left(\chi^{3}+\chi-2\right)\left[\chi^{2} \sec ^{-1}(\chi)-\left(\chi^{2}-1\right)^{\frac{1}{2}}\right]^{2}\left(\chi^{2}-1\right)^{-3} \\
\chi=1+\frac{9}{64} W e+O\left(W e^{2}\right)
\end{gathered}
$$

The correlation recently proposed by Loth (2008) for bubbles rising freely in a uniform flow by compiling a large data base with $R e$ over the range $0.2-5000$ :

$$
\frac{1}{\chi}=1-\left(1-E_{\text {min }}\right) \tanh \left(c_{E} W e\right)
$$

with

$$
E_{\min }=0.25+0.55 \exp (-0.09 R e)
$$

and

$$
c_{E}=0.165+0.55 \exp (-0.3 R e)
$$

has also been reported in this figure. Our data are in between the complete and small-deformation expressions of Moore (1965) at low to moderate Weber numbers, but rapidly these expressions respectively under- and over-estimate the measured aspect ratios. Referring to Loth (2008), this can be explained by the fact that the fore-aft symmetry of the bubble is progressively broken as $\chi$ increases and that the theories do not account for the open wake which then develops. In comparison, equation 5.7, which asymptotically tends to equation 5.6 at small $W e$, well describes our experimental results over the whole range of $(W e, R e)$ explored, except at high deformation rates $(\chi>2.4, W e>6))$. $E_{\text {min }}$ stands for the inverse of the upper aspect ratio that can be reached as the bubble deforms. It generally corresponds to spherical cap bubbles. Before reaching this shape, the increase in $\chi$ starts to saturate when the boundary layer around the bubble separates and a separated wake appears. Here, this situation is clearly observed for the oil such that with $\log (M o)=-3.8$. The beginning of the saturation appears earlier than it is predicted by equation 5.7. Apart from that case, we can expect, by referring to the $R e-\chi$ phase diagram of Blanco and Magnaudet (1995) established for uniform flows, the wakes to remain attached for all other flow conditions. Over the range $\chi \in[1 ; 2.4], W e \in[1 ; 6]$, a least-square fit of the results shows that the aspect ratios are well approximated by a simple relationship of the form

$$
\chi=1+\frac{9}{64} W e+\frac{3}{250} W e^{2}+O\left(W e^{3}\right)
$$

which is almost the second-order expansion of equation 5.7 within the limit of small $R e, W e$. 


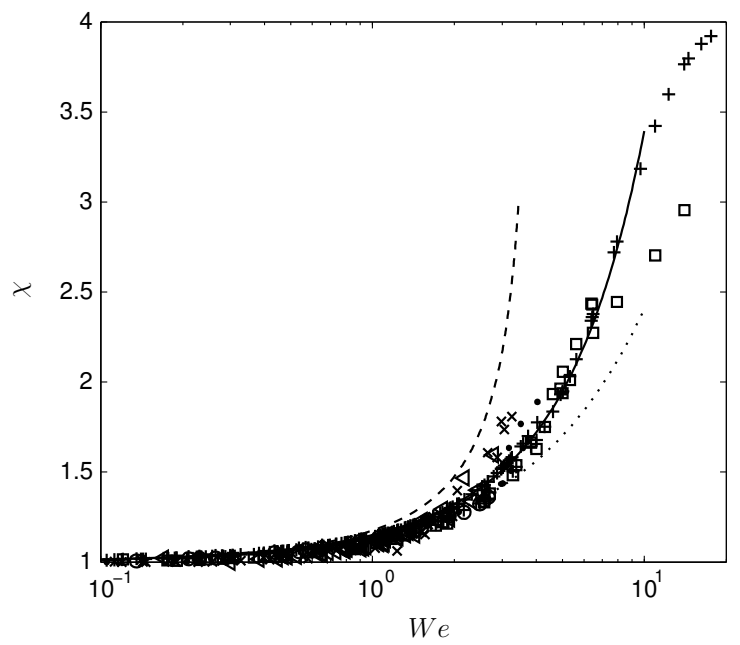

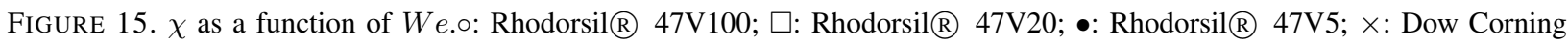
$200 \AA 1 \mathrm{cst} ; \triangleleft$ : Dow Corning 200® 0.65cst. Dotted line: equation 5.6; dashed line: equation 5.5. +: Loth (2008)'s values for $\chi$ for the corresponding $(R e, W e)$ values; solid line: equation 5.8 .

The fact that the aspect ratio does not depend on Ro seems consistent with the simplifications introduced in subsection 5.1. The shape of the deformed bubbles is treated as ellipsoid, even in cases where deformation becomes asymmetric (the highest viscous silicon oils). In reality, the deformed shape is more complex. It is a combination of symmetric and asymmetric surface deformation modes, which are often modeled in potential flow in terms of spheroidal surface harmonics (Lamb 1934). It is reasonable to think that the first symmetric deformation mode that we consider here and characterize by $\chi$, will essentially depend on $W e$, while the other asymmetric modes will be more influenced by Ro.

Contrary to what happens with a spherical bubble, an ellipsoidal bubble experiences non-zero torque when immersed in a non-uniform flow like a $S B R H$. Indeed the bubble being clean, all the forces are locally normal to the surface. If the flow is non-axisymmetric, their resultant is not directed toward the center of inertia. As a result, bubbles will orient so that the torque becomes nil. This is why an angle $\alpha$ between the direction of the flow and the minor axis is observed. In uniform flows, the torque is nil when this angle is zero, except when the clean ellipsoidal bubble path is instable, that is for a Re number greater about 600 (Duineveld 1995; Veldhuis et al. 2008). In that case, zig-zagging or spiraling bubbles undergo shape oscillations coupled to vortex shedding and bubble rotates periodically about its minor axis in response to the new rising direction (Lunde and Perkins 1998). This does not concern the bubbles studied here since their $R e$ is far lower. The angle is constant in time, meaning that no instability takes place. Figure 16 shows how this angle varies with the Ro number. For the two highest Morton numbers (Dow Corning 200ß 0,65 cst, 1cst) the ellipsoid minor axis is nearly aligned with the direction of the flow. This corresponds to situations where $R o>5$ (figure 3), thus the non-dimensional local shear rate $S r=R o^{-1}$ is small. The angle is then typically of the order of one degree, which is close to the observations of Tomiyama et al. (2002) in a linear shear flow, under similar deformations and shear rates. The same kind of result has been obtained numerically at high Reynolds numbers by Adoua (2007) for ellipsoidal clean bubbles in a linear shear flow. The author shows that this small angle corresponds to the stable solution for the position of the bubble. It depends both on the aspect ratio and the local shear rate and is shown to increase as $\mathrm{Sr}^{1 / 3}$. Here, the angle also increases in absolute value as $R o$ decreases. This increase becomes more pronounced at lower Mo numbers, when fluids are more viscous (Rhodorsil $囚 47 \mathrm{~V} 5,47 \mathrm{~V} 20$ ) and bubbles still approximately ellipsoidal. For asymmetric bubbles and the few spherical cap ones, $\alpha$ takes on large absolute values. This is due among other things to the fact that these bubbles frequently have an equilibrium position little or well above the horizontal line. But in that case the aspect ratio, like the angle, are not really adapted to characterize the real shape. Nevertheless, angles for the non-ellipsoidal bubbles are somehow in the continuity of those for ellipsoidal ones.

\subsection{Drag forces}

As can be seen in figure 17, drag is more important for deformed ellipsoidal bubbles than for the equivalent spherical ones, whatever the Mo number. This expected trend can be attributed to two effects: an increase of pressure drag linked to an increase of the apparent cross section and an increase of friction drag in the curved boundary layer developing 


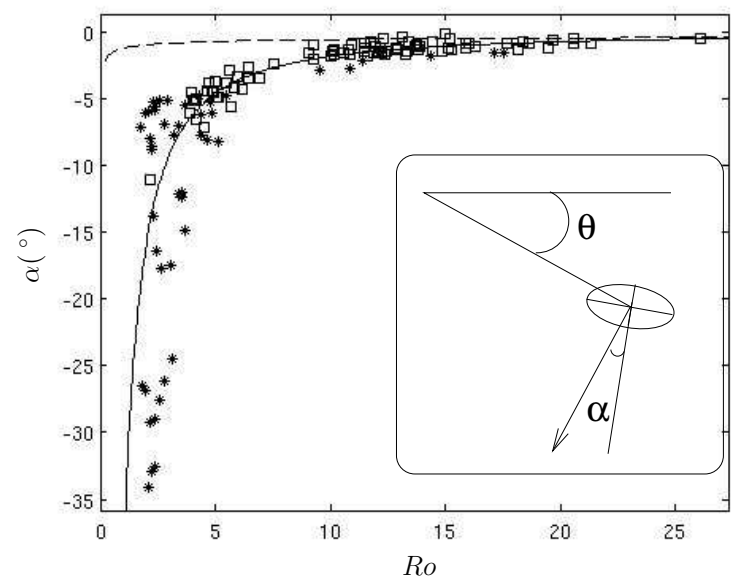

FIGURE 16. $\alpha\left({ }^{\circ}\right)$ as a function of $R o . \square$ : ellipsoidal bubbles, $*$ : asymmetric or spherical cap bubbles. Solid line: $-\exp (11 / 3) R^{-4 / 3}$, dashed line: $-1.2 R o^{-1 / 3}$.

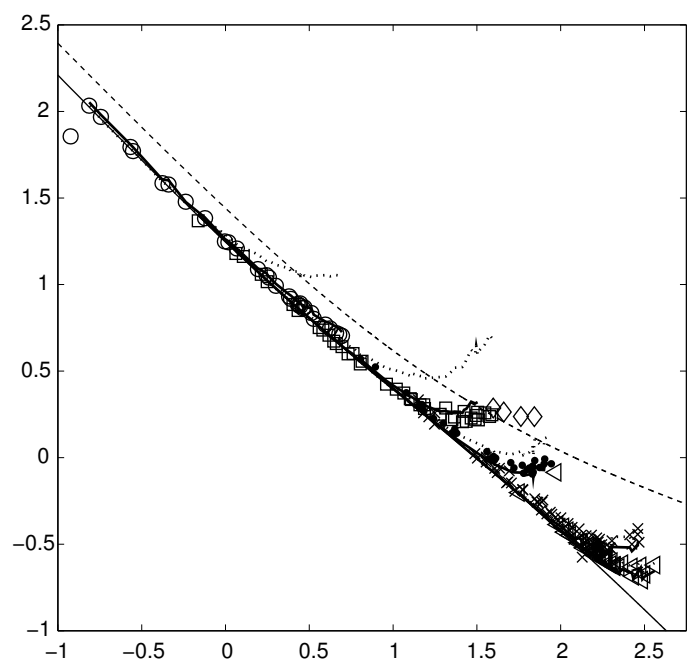

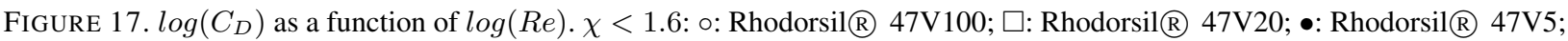
$\times$ : Dow Corning $200 ® 1 \mathrm{cst} ; \triangleleft$ : Dow Corning $200 ® 0.65 \mathrm{cst} . \chi \geq 2$ : $\diamond$. Solid line: drag curve for clean spherical bubbles in a uniform flow $\left(C d_{\text {uniform }}\right.$ ) (Mei et al. 1994); dashed line: drag curve for solid spheres in a uniform flow (Schiller and Nauman 1933 see Clift et al. (1978)); thick solid lines: equation 5.15; dotted line: equation 5.16, increase of drag expected from the theory of Moore (1965).

around the bubble (Moore 1965; Blanco and Magnaudet 1995). The relative importance of the two contributions varies both with $R e$ and $\chi$. We note that the increase of the drag with the Rhodorsil@ 47V100 oil (low Re, low $M o$ ) remains weak. This can be explained, by the particular shape of the bubble in this highly viscous oil and the fact that the aspect ratio $(\chi<1.4)$ does not reach sufficient values to enhance the two components of drag (see for instance picture (d) in figure11). For practical applications, it is convenient to represent our experimental results with an analytical drag law as simple as possible. Such a law must of course be consistent with the asymptotic behavior of the drag coefficient for distorted bubbles at high and low Reynolds numbers, in the absence of rotation. The first case is correctly described by the theory of Moore (1965), while the second can be deduced from the extension of Oseen's correction to the creeping flow around a sphere. Brenner (1961) found the general form of the Oseen's drag for a particle of arbitrary shape, and Breach (1961) gave the exact expression for a solid oblate ellipsoid, but these works are not adapted to a bubble subject to the shear-free condition at the interface. In the limit of low but finite $R e$, expressions for the drag and shape 
of a drop of arbitrary density and viscosity were derived by Taylor and Acrivos (1964) using matched asymptotic expansions, combined with the result of Brenner and Cox (1963). The case where the density and viscosity tend to zero corresponds to a clean bubble and the aspect ratio and the drag coefficient are then given by:

$$
\begin{gathered}
\chi=1+\frac{5}{32} W e+O\left(W e^{2}\right) \\
C_{D, T \& A}=1+\frac{16}{R e}\left[1+\frac{R e}{8}+\frac{W e}{12}+O\left(\operatorname{Re}^{2} \operatorname{Ln}(R e)\right)\right]
\end{gathered}
$$

It follows that the effect of the deformation on the drag is accounted for by a correction of the order of $W e$, which remains small as long as the bubble's shape does not depart too much from the sphere. This is the case in the present experiments, since the low Reynolds number range is associated with small Weber numbers. Combining equations 5.10 and 5.9, yields the drag coefficient at low but finite $R e$ :

$$
C_{D, T \& A}=1+\frac{16}{R e}\left[1+\frac{R e}{8}+\frac{8}{15}(\chi-1)\right]
$$

which tends to Oseen's drag as $\chi \rightarrow 1$. The drag law of Moore was calculated by assuming that the viscous flow around the bubble was confined in a thin viscous sublayer which remained attached. It is expressed as:

$$
C_{D, \text { Moore }}=\frac{48}{R e} G(\chi)\left[1-\frac{2.21 H(\chi)}{R e^{\frac{1}{2}}}\right]
$$

where $\mathrm{G}(\chi)$ sand $\mathrm{H}(\chi)$ are geometrical factors calculated by Moore (1965), and which can be accurately approximated by (Loth (2008))

$$
G(\chi) \simeq 0.1287+0.4256 \chi+0.4466 \chi^{2} ; H(\chi) \simeq 0.8886+0.5693 \chi-0.4563 \chi^{2}
$$

The simplest starting point to find a drag law valid for all the Re spanned here is that proposed by Mei et al. (1994) to represent the drag coefficient of a spherical bubble moving in a quiescent liquid, which matches the two asymptotic limits and fits the numerical simulations in the intermediate range of Reynolds numbers. A modification of the formula to account for the deformation reads in:

$$
C_{D}(\chi)=\frac{16}{R e}\left[\frac{1+\frac{8}{15}(\chi-1)+0.015(3 G(\chi)-2) R e}{1+0.015 R e}+\left[\frac{8}{R e}+\frac{1}{2}\left(1+\frac{3.315 H(\chi) G(\chi)}{R e^{\frac{1}{2}}}\right)\right]^{-1}\right]
$$

The prefactor 0.015 and the exponent 1 of Re in the first term have been determined to give the best fit of the experimental data throughout all the whole range of Re spanned. The above expression retrieves Moore's drag law at large Reynolds numbers, and Taylor and Acrivos's drag law when Re and $\chi$ are small. A last step is necessary to represent the effect of rotation. Adoua (2007) found that for a linear shear flow, the drag should be corrected by a multiplicative factor function of the shear parameter. Following the same line, we simply use the correction in equation 4.2 obtained for a spherical bubble, which yields :

$$
C_{D}(\chi)=\frac{16}{R e}\left[\frac{1+\frac{8}{15}(\chi-1)+0.015(3 G(\chi)-2) R e}{1+0.015 R e}+\left[\frac{8}{R e}+\frac{1}{2}\left(1+\frac{3.315 H(\chi) G(\chi)}{R e^{\frac{1}{2}}}\right)\right]^{-1}\right]\left(1+\frac{0.3}{R o^{\frac{5}{2}}}\right)
$$

Equation 5.15 is plotted in a solid line in figure 17. It predicts well the experimental trends in the range $\chi<2$ where deformation is not too high and bubbles approximately ellipsoidal. We see that for the oil with the intermediate viscosity (Rhodorsil@ 47V20), the drag appears to saturate and no longer increases as rapidly as before. This is because bubbles are asymmetrical - even spherical cap - that probably the boundary layer separates and a separated wake appears. The increase of drag expected from the theory of Moore (1965) and calculated as:

$$
C_{D}(\chi)=C_{D, M e i}+\left(C_{D, M o o r e}(\chi)-C_{D, \text { Moore }}(1)\right)
$$

is also plotted in a dotted line in the same figure. It reproduces well the trends at high $R e$ (high $R o$ ) but unsurprisingly, predict increases that are all the more over-estimated as the oil are more viscous and the $R e$ numbers low. The modified drag law 5.15 can be expressed as a function of $W e$ number from equation 5.8 (or equation 5.9 when $R e$ is low) and the $W e$ as a function of $(R e, M o)$ from equation 5.4. 

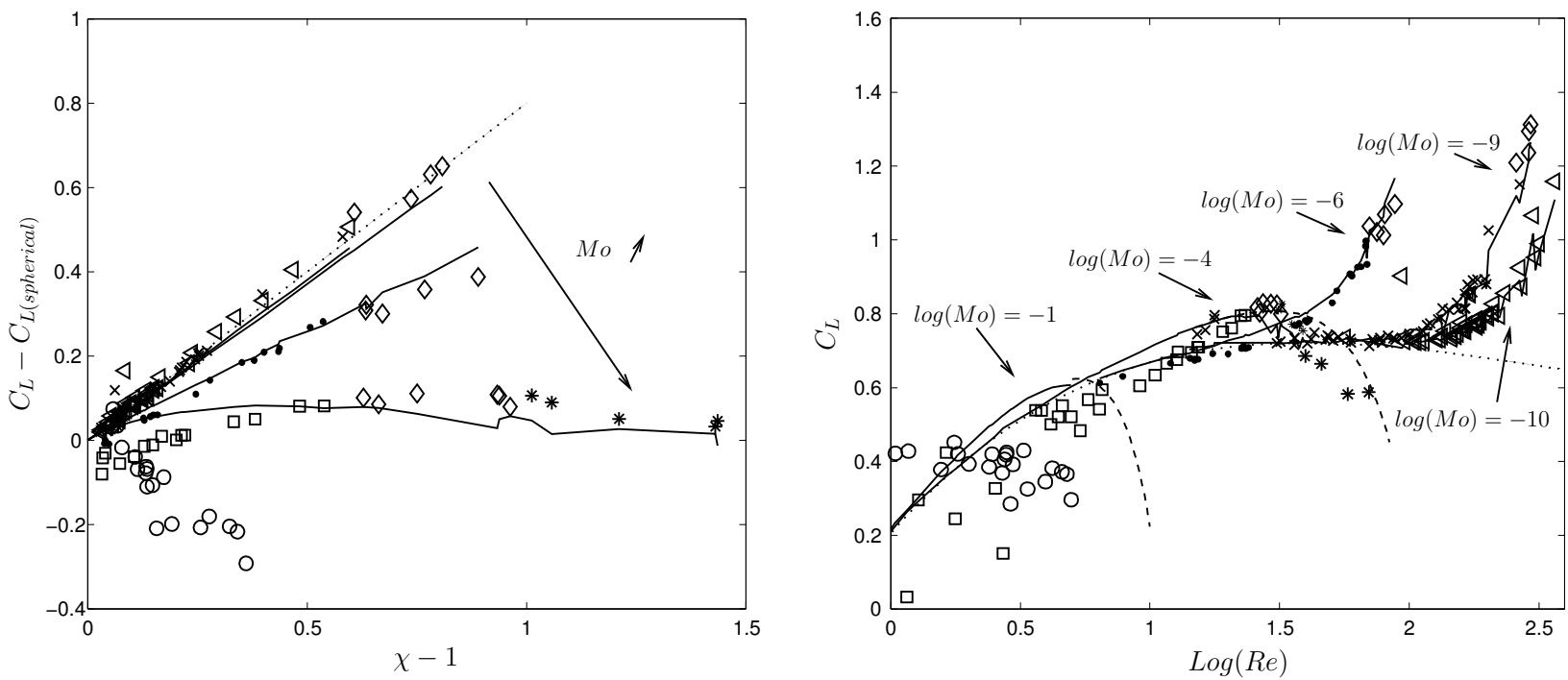

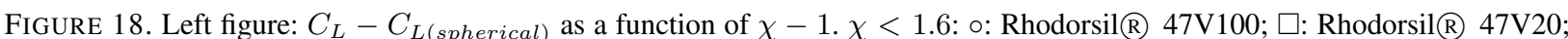

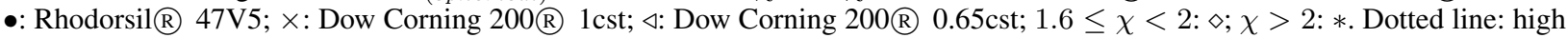
Reynolds limit $0.8(\chi-1)$; solid line: $0.8(\chi-1)-1.3(\chi-1)^{3 / 2} /\left(1+0.004 R e^{3 / 2}\right)$. Right figure: $C_{L}$ as a function of $\log (R e)$. Same symbols. Dotted line: equation 4.4; solid line: equation 5.17; dashed line: extrapolated trends at high deformation rates, as calculated by equation 5.17 together with equation 5.8 and equation 5.4.

\subsection{Lift forces}

Lift coefficients for deformed bubbles are plotted in figure 18 versus $R e$ number on the right, and the aspect ratio on the left. Since they are calculated assuming an added mass coefficient valid for ellipsoids (equation 2.5), they can be considered as unbiased by this restriction for $\chi<2.5-3$ (Fig. 15). For the less viscous fluids (viscosities: $5 \mathrm{cSt}, 1 \mathrm{cSt}$ and $0.65 \mathrm{cSt}$ ), the deformation of the bubble from the spherical shape to the ellipsoidal shape increases the lift. The higher the We number, the higher the lift coefficient. For $\nu=20 \mathrm{cSt}$, an increase followed by a decrease is observed when the We number is increased. For $\nu=100 \mathrm{cSt}$, the behavior is not so easy to analyze. Nevertheless, it can be mentioned that in such a fluid the increase in $W e$ is linked to a decrease in the lift coefficient.

$$
C_{L}=C_{L(\text { spherical })}+0.8(\chi-1)-1.3 \frac{(\chi-1)^{\frac{3}{2}}}{1+0.004 R e^{3 / 2}}
$$

fits the variation of the lift coefficient with the aspect ratio $\chi$ and with the $R e$ number for the three lower viscosities ( $5 \mathrm{cSt}, 1 \mathrm{cSt}$ and $0.65 \mathrm{cSt}$ ) closely. The lift increase is reproduced well. For the higher viscosity $(20 \mathrm{cSt}$ ), the increase followed by the decrease is also predicted but with a slight shift with the experimental results. The irregularities of the solid lines corresponding to equation 5.17 in figure 18 are linked to the experimental dispersion in $\chi$ and $R e$.

Physically, the second term in equation 5.17 expresses the increase of $C_{L}$ with the aspect ratio within the limit as $R e$ tends to infinite. It is close to the theoretical solution obtained for ellipsoidal bubbles in a weak inviscid shear flow by Naciri (1992), who showed that this solution closely follows the evolution of the added mass coefficient. The last term is a Re-dependent correction which is suggested by the recent simulations by Adoua (2007), Adoua et al. (2009) for oblate bubbles in a weakly viscous linear shear flow. For clean bubbles, the lift force results from the integration of the pressure and the normal viscous stress at the interface. The simulations prove that for $R e>100$, the pressure contribution is dominant and that the evolution of lift with deformation is directly linked to the asymmetric distribution of pressure at the surface of the bubble. This asymmetric distribution is linked to the top-bottom asymmetry of the boundary layer generated at the bubble surface by the inhomogeneity of the base flow. It induces a viscous pressure correction that contributes to the lift and behaves as $R e^{-3 / 2}$, thus justifying the form of the correction term. Here, the denominator is almost the same as in Adoua et al. (2009), while the numerator is the one which provides the best fit of our data. A contribution for the normal viscous stress should also be included for $R e$ below 100, unfortunately there are not enough data in that range to find the Re-dependence of this contribution.

It can be noticed that there is no discontinuity (rupture) in the evolution of the lift coefficients as bubbles become asymmetric. At first order, they follow the trends (dashed lines in right figure 18) obtained for highly deformed el- 
lipsoidal bubbles from equation 5.17, 5.8 and 5.4. With the oil of viscosity $20 \mathrm{cSt}$ an inversion of the lift force seems possible as $R e$ will increase further. Such a reversal of the lift force has been observed in some experiments in linear shear flow by Kariyasaki (1987), Tomiyama et al. (2002) and is predicted by Adoua et al. (2009). The experiments of Tomiyama et al. (2002) were performed in mixtures of water and glycerine over the range of parameters: $M o \in\left[10^{-5.5} ; 10^{-2.8}\right]$, equivalent $R e \in[5 ; 80], E_{o} \in[1.4 ; 5.7]$, and $S \leq 0.1$. According to this work, a change of lift sign can be expected when $E o>4$, the evolution of the lift coefficient being then described by the empirical relationship:

$$
C_{L}=0.00105 E o^{3}-0.0159 E o^{2}-0.0204 E o+0.474
$$

The oils which have $R e$ typically in the range $\in[5 ; 80]$ are the Rhodorsil $(47 \mathrm{~V} 20$, and the Rhodorsil $\AA$ 47V5. In the first case, $E_{O} \in[0.2 ; 8.7]$ and one should expect from equation 5.18 a reversal of the lift in a linear flow, while here, the lift decreases but does not change sign. This difference comes from the fact that the lift coefficients in Tomiyama et al. (2002), are initially equal to 0.3 , thus much lower than ours over this range of $R e$. In the second case, Eo $\in[0.2 ; 2.8]$ so that a stabilization of the lift around a constant value 0.3 is predicted in a linear flow, while it clearly increases here with deformation. Comparing the two flow situations must therefore be considered with great caution.

According to the simulations of Adoua et al. (2009), the inversion of the lift is due to competition between the vorticity generated at the interface and the upstream vorticity tilted around the bubble. It occurs when the aspect ratio reaches a critical value, function of the non dimensional shear rate. Here the reversal, if it takes place, will correspond to spherical cap bubbles or even more deformed ones. The reason is that, unlike what can be done numerically, it is not possible to generate ellipsoidal bubbles of large $R e$ numbers with a large aspect ratio for a given $M o$ number with the silicon oils we use. The surface tension is much lower than that of water (typically 3.5 to 4.5 times smaller). Subsequently, as size increases, bubbles depart from the ellipsoidal shape rapidly. Hence, it is not possible to expect an inversion of lift with an ellipsoidal bubble, whereas it appears to be possible with a spherical cap one for some given $M o$ numbers. Finally bubbles are free to orientate unlike in the calculations, which could be another source of differences. Adoua (2007) compared the value of the lift and drag coefficients obtained when the angle is nil, and the angle is equal to the equilibrium value for which the torque exerted on the bubble in a linear shear flow is nil. He found that the differences induced by the bubble inclination are of the order of a few percent for $\operatorname{Re}=1000$ and $\chi \in[0.2 ; 2]$, but become significant for $R e=400$ and $\chi=2.5$, with a possible consequence on the transition where the lift sign changes.

\section{Conclusion}

This article is an extension of our previous work on contaminated bubbles (Rastello et al. 2009). The purpose was to measure the drag and lift coefficients on clean spherical and deformed bubbles in a $S B R H$ flow. To ensure the bubbles' cleanliness, experiments have been performed with five silicon oils, giving a large panel of viscosities and Mo numbers. In the regime where bubble are spherical, the dependence of drag coefficients on Re number is very close to that of clean bubbles rising in uniform flows. This validates the fact that the shear-free condition at the interface is satisfied. The drag coefficients are found to increase when the $R o$ number decreases. This increase varying as $R o^{-\frac{5}{2}}$ is comparatively smaller than that calculated at much higher $R e$ for linear shear flows (Legendre and Magnaudet 1998). Regarding the lift coefficients, they coincide with the ones obtained numerically by Magnaudet and Legendre (1998) for clean spherical bubbles in a $S B R H$. A fit of both their and our results has been proposed. It covers the four decades of all the results. The excellent agreement brings additional evidence that the higher lift coefficients reported in Rastello et al. (2009) were due to the contamination of the interfaces (the liquid is not free to slip along the bubble surface any more) and the ensuing spinning of the surface layer. No influence on the Ro number has been revealed, which is consistent with the numerical results. Special attention was paid to the low Re numbers. For very low values of the Re number, measurement of the lift coefficient is shown to be problematic, so that a change of lift sign over that range, if it takes place, is hardly detectable. Strongly decreasing the framing rate allowed some bubbles to be filmed as they shrank. The drag and lift measured from successive bubble time positions turns out to follow the curves of nonshrinking bubbles, indicating that the contracting process is quasi-static. In the regime where bubbles deform, the shape is generally close to an ellipsoidal spheroid and becomes more asymmetric for the most viscous oils. The transition between the spherical and ellipsoidal shapes in the $S B R H$ flow has been determined by revisiting the well-known Clift et al. (1978) diagram. The transitions between the two regimes occur on average at the same $(R e, E o)$ values as for bubbles ascending in a uniform flow. Whatever the silicon oil, the aspect ratio of the ellipsoidal bubbles approximately increases with $W e$ number following the recent correlation by Loth (2008). At low and moderate deformation rates, 
the increase is in between the complete theory of Moore (1965) for ellipsoidal bubbles, and its small-deformations expansion (Moore 1959). Under the action of the torque exerted by the flow, deformed bubbles orient their small axis at an angle of a few degrees to the incoming flow, comparable to that calculated in linear shear flows (Adoua et al. 2009). However this angle becomes greater at low Mo or Ro numbers. At the same equivalent diameter, the drag coefficient is higher for ellipsoidal bubbles than for spherical ones, the difference being both a function of the aspect ratio or, which is equivalent, of the $W e$ number and the $R e$ number. The increase of drag is well predicted by the theory of Moore (1965) at high $R e$ and high $R o$, but is over-estimated by this theory at low $R e$, as expected. A modified expression of the Mei et al. (1994) drag law that reproduces the experimental trends over the whole range of $R e$ numbers has been proposed. It retrieves the drag law of Moore (1965) at high Re, that of Taylor and Acrivos (1964) at low but finite $R e$, and includes both the effects of deformation and rotation. The behavior of the lift coefficients also depends both on the aspect ratio and $R e$ number. For high $R e$ numbers (less viscous oils) the lift coefficients increase almost linearly with the aspect ratio, in line with the inviscid theory, whereas in an intermediate range (more viscous oils) the increase is followed by a decrease at a high deformation rate. However, we never reached the conditions for reversal of lift to occur. An approximate relationship that reproduces the lift tendencies rather well over the range of parameters investigated has been proposed. It includes a shear-induced viscous pressure correction of the form suggested by the simulations of Adoua et al. (2009). This correction arises from the top-bottom asymmetry of the boundary layer generated at the bubble surface by the inhomogeneity of the base flow and is supposed to behave like $R e^{-\frac{3}{2}}$, as in a linear shear flow.

The authors would thank Mr Magand of Caldic society, and Dow Corning corporation, for providing them the

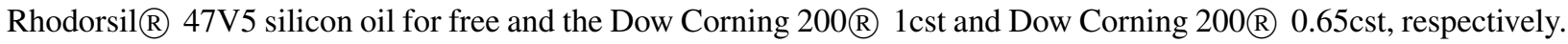
They also thank C. Colin for her useful advice on silicon oils.

\section{REFERENCES}

R. Adoua, D. Legendre, and J. Magnaudet. Reversal of the lift force on an oblate bubble in a weakly viscous shear flow. J. Fluid Mech., 628:23-41, 2009.

S.R. Adoua. Hydrodynamique d'une bulle déformée dans un écoulement cisaillé. PhD thesis, Institut National Polytechnique de Toulouse, 2007.

T.R. Auton. The lift force on a spherical body in a rotational flow. J. Fluid Mech., 183:199-218, 1987.

P. Bagchi and S. Balachandar. Effect of free rotation on the motion of a solid sphere in linear shear flow at moderate Re. Phys. Fluids, 14(8):2719-2737, 2002.

A. Blanco and J. Magnaudet. The structure of the axisymmetric high-Reynolds number flow around an ellipsoidal bubble of fixed shape. Phys. Fluids, 7:1265-1274, 1995.

J.J. Bluemink, D. Lohse, A. Prosperetti, and L. Van Wijngaarden. A sphere in a uniformly rotating or shearing flow. J. Fluid Mech., 600:201-233, 2008.

J.J. Bluemink, D. Lohse, A. Prosperetti, and L. Van Wijngaarden. Drag and lift forces on particles in a rotating flow. J. Fluid Mech., 643:1-31, 2010.

D.R. Breach. Slow flow past ellipsoids of revolution. J. Fluid Mech., 10:303-314, 1961.

H. Brenner. The oseen resistance of a particle of arbitrary shape. J. Fluid Mech., 11:604-610, 1961.

H. Brenner and R.G. Cox. The resistance to a particle of arbitrary shape in translational motion at small reynolds numbers. J. Fluid Mech., 17:561-595, 1963.

F. Candelier, J.-R. Angilella, and M. Souhar. On the effect of the Boussinesq-Basset force on the radial migration of a Stokes particle in a vortex. Phys. Fluids, 16(5):1765-1776, 2004.

R. Clift, J.R. Grace, and M.E. Weber. Bubbles, Drops and Particles. Academic, 1978.

A. W. G. de Vries, A. Biesheuvel, and L. van Wijngaarden. Notes on the path and wake of a gas bubble rising in pure water. Int. J. Multiphase Flow, 28:1823-1835, 2002a.

J. de Vries, S. Luther, and D. Lohse. Induced bubble shape oscillations and their impact on the rise velocity. The European Physical Journal B, 29(3):503-509, 2002b.

P.C. Duineveld. The rise velocity and shape of bubbles in pure water at high Reynolds number. J. Fluid Mech., 292:325-332, 1995.

E.A. Ervin and G. Tryggvason. The rise of bubbles in a vertical shear flow. J. Fluid Eng., 119:443, 1997.

T. Gotoh. Brownian motion in a rotating flow. J. Stat. Phys., 59:371-402, 1990.

J.R. Grace, T. Wairegi, and T.H. Nguyen. Shapes and velocities of single drops and bubbles moving freely through immiscible liquids. Trans. Instn. Chem. Engrs, 54:167-173, 1976.

E.Y. Harper and I.D. Chang. Maximum dissipation resulting from lift in a slow viscous shear flow. J. Fluid Mech., 33:209-225, 1968.

A. Kariyasaki. Behavior of a single gas bubble in a liquid flow with a linear velocity profile. In Proceedings of ASME-JSME Thermal Engng Joint Conf., Honolulu Hawaii, pages 261-267, 1987. 
H. Lamb. Hydrodynamics 6th edition. Dover, 1934.

D. Legendre and J. Magnaudet. The lift force on a spherical bubble in a viscous linear shear flow. J. Fluid Mech., 368:81-126, 1998.

V.G. Levich. Physicochemical Hydrodynamics. Prentice Hall, 1962.

E. Loth. Quasi-steady shape and drag of deformable bubbles and drops. Int. J. Multiphase Flow, 34(6):523-546, 2008.

K. Lunde and R.J. Perkins. Shape oscillations of rising bubbles. Applied Scientific Research, 58:387-408, 1998.

J. Magnaudet and I. Eames. Dynamics of high re bubbles in inhomogeneous flows. Annu. Rev. Fluid Mech., 32:659-708, 2000.

J. Magnaudet and D. Legendre. Some aspects of the lift force on a spherical bubble. Appl. Sci. Res., 58:441-461, 1998.

R. Mei, J. Klausner, and C. Lawrence. A note on the history force on a spherical bubble at finite Reynolds number. Phys. Fluids, 6:418-420, 1994.

D.W. Moore. The rise of a gas bubble in a viscous liquid. J. Fluid Mech., 6:113-130, 1959.

D.W. Moore. The velocity rise of distorted gas bubbles in a liquid of small viscosity. J. Fluid Mech., 23:749-766, 1965.

A. Naciri. Contribution à l'étude des forces exercées par un liquide sur une bulle de gaz: portance, masse ajoutée et interactions hydrodynamiques. PhD thesis, Ecole Centrale de Lyon, 1992.

M. Rastello, J.-L. Marié, N. Grosjean, and M. Lance. Study of bubble's equilibrium in a rotating flow. In 6th Int. Conf. Multiphase Flows, Leipzig, July 9-13, 2007.

M. Rastello, J.L. Marié, N. Grosjean, and M. Lance. Drag and lift forces on interface-contaminated bubbles spinning in a rotating flow. J. Fluid Mech., 624, 2009.

P.G. Saffman. The lift force on a small sphere in a slow shear flow. J. Fluid Mech., 22:385-400, 1965.

A. Serizawa, I. Kataoka, and I. Michiyoshi. Turbulence structure of air-water bubbly flow ii. Int. J. Multiphase Flow, 2:235-246, 1975.

F. Takemura, S. Takagi, J. Magnaudet, and Y. Matsumoto. Drag and lift forces on a bubble rising near a vertical wall in a viscous liquid. J. Fluid Mech., 461:277-300, 2002.

F. Takemura, J. Magnaudet, and P. Dimitrakopoulos. Migration and deformation of bubbles rising in a wall-bounded shear flow at finite reynolds number. J. Fluid Mech., 634:463-486, 2009.

T.D. Taylor and A. Acrivos. On the deformation and drag of a falling viscous drop at low Reynolds number. J. Fluid Mech., 18: 466-476, 1964.

M. Teague. Image analysis via the general theory of moments. J.of.Opt.S., 70(8):920-930, 1980.

A. Tomiyama, H. Tamai, I. Zun, and S. Hosokawa. Transverse migration of single bubbles in simple shear flows. Chem.Engng Sci., 57:1849-1859, 2002.

E.A. van Nierop, S. Luther, J.J. Bluemink, J. Magnaudet, A. Prosperetti, and D. Lohse. Drag and lift forces on bubbles in a rotating flow. J. Fluid Mech., 571:439-454, 2007.

C. Veldhuis, A. Biesheuvel, and L. Van Wijngaarden. Shape oscillations on bubbles rising in clean and tap water. Phys. Fluids, 4 (20):040705-1-040705-12, 2008.

L. Wakaba and S. Balachandar. History force on a sphere in a weak linear shear flow. Int. J. Multiphase Flow, 31(9):996-1014, 2005.

R. Zenit and J. Magnaudet. Path instability of rising spheroidal air bubbles: a shape-controlled process. Phys. Fluids, 20:061702, 2008. 